\title{
Adaptive finite-time tracking control for a class of switched nonlinear systems with unmodeled dynamics
}

\author{
Jun Mao, Zhengrong Xiang*, Shipei Huang \\ School of Automation, Nanjing University of Science and Technology, \\ Nanjing, 210094, Peoples Republic of China
}

\begin{abstract}
In this paper, an adaptive tracking control scheme is proposed for a class of switched nonlinear systems with state and input unmodeled dynamics. The unmodeled dynamics are dealt with by introducing a first-order filter and a dynamic signal. K-filters are used to estimate the unmeasured states, and the dynamic surface control (DSC) technique is employed to construct the controller to avoid the explosion problem of complexity. By choosing an appropriate common Lyapunov function, the boundedness of all closed-loop signals is proved, and the tracking error can converge to a small neighbourhood of zero in finite time under arbitrary switchings. Finally, a simulation example is provided to show the feasibility and validity of the proposed method.
\end{abstract}

Keywords: Switched systems, nonlinear systems, unmodeled dynamics, K-filters, dynamic surface control

\section{Introduction}

Over the past years, switched systems have become an emerging hot research topic due to their board applications in control fields, such as spacecraft control [1], vehicle control $[2,3]$ and so on. Switched system means a hybrid system that is composed of a family of continuous-time and discrete-time subsystems and a rule orchestrating the switching between the subsystems. Stabilization and tracking are fundamental problems in the research field of switched nonlinear systems [4-20]. Many significant methods have been proposed to solve these problems, such as common Lyapunov function method, multiple Lyapunov function method and dwell-time approach [6, 14, 15, 17, 18, 20]. For example, common Lyapunov function method were employed to solve the tracking control problem of switched nonlinear systems in strict feedback form $[14,17,18]$. Adaptive tracking control for switched nonlinear systems in lower-triangular form was investigated in [6] by exploiting multiple Lyapunov function method. By using the dwell-time approach, adaptive control for uncertain switched

\footnotetext{
*Corresponding author

Email address: xiangzr@mail.njust.edu.cn (Zhengrong Xiang)
} 
nonlinear systems was studied in [15, 20], where fuzzy sets [21, 22] and neural networks [19, 23-25] are used to approximate unknown nonlinearities.

As is well known, unmodeled dynamics widely exist in many practical nonlinear systems, which can severely degrade the system performance. Therefore, how to handle unmodeled dynamics is a meaningful topic when one investigates the system stability. Generally speaking, unmodeled dynamics include state unmodeled dynamics [5, 6, 15, 20, 25-32] and input unmodeled dynamics [33-38]. State unmodeled dynamics denote the parts of invalid modeling during the parameterization, a few approaches were proposed to handle the adverse effects caused by them. In [5, 6, 20, 26, 27, 29-32], the state unmodeled dynamics were dominated by introducing available dynamic signals. In [15, 28], several specific Lyapunov functions were selected to remove the state unmodeled dynamics. On the other hand, input unmodeled dynamics mean modeling errors or external disturbances act upon the controller. In [33-38], a first-order filter was introduced to generate a dynamic signal to overcome the input unmodeled dynamics, which were of relative degree zero and minimum-phase.

In recent years, finite-time stabilization and finite-time tracking have drawn considerable attention due to theirs practical importance [1, 16-18, 39-42]. The aim of finite-time stabilization or tracking is to design the control law to make system states or tracking errors converge to the origin or the small neighbourhood of it in finite time. In [39], the problem of global finite-time stabilization for a class of stochastic nonlinear systems was solved. In [16-18], finite-time stabilization was studied for several classes of switched nonlinear systems in strict feedback form. Finite-time tracking and stabilization control for spacecraft systems were respectively investigated in [1] and [40]. In [41, 42], adaptive finite-time tracking and stabilization control schemes were respectively proposed for multi-agent and autonomous systems. However, finite-time tracking control for switched nonlinear systems with unmodeled dynamics was not investigated until now, which motivates the present study. The main contributions of this paper are summarized as follows:

(i) An adaptive finite-time tracking control scheme is proposed for a class of switched nonlinear systems with unmeasured states and unmodeled dynamics. K-filters are used to estimate the unmeasured states, and a filter is introduced to counteract the influence of input unmolded dynamics. Moreover, dynamic surface control (DSC) is used to overcome the limitation of "explosion of complexity".

(ii) Compared with previous results [29-31, 33-35], the restrictions on the control coefficients and the reference signals are relaxed. In this paper, the upper bound of control coefficient is unknown and the second derivative of the reference signal is not required to be bounded.

(iii) An output feedback controller and adaptive laws are constructed to guarantee that the tracking error can converge to a small neighbourhood of zero in finite time rather than in infinite time presented in [20, 29-31, 33-35].

The rest of this paper is organized as follows. In Section 2, the problem statement and preliminaries are given. In Section 3, system parameterization and K-filters design are presented. In Section 4, a control scheme is developed for switched systems by using the DSC 
technique. Section 5 gives stability analysis. Simulation results are presented in Section 6 . Section 7 summarizes the main conclusions.

Notations: $R^{+}$denotes the set of all non-negative real numbers; $R^{n}$ denotes the real $n$ dimensional space; $R^{m \times n}$ denotes the real $m \times n$ dimensional matrix; $R_{\text {odd }}^{+} \triangleq\{q \in R: q>0$ and $q$ is a ratio of odd integers $\} ; e_{i}, i=1,2, \cdots, n$, denotes the $n$-dimensional vector with the $i$ th element being one, and other elements being all zeros; $K$ function denotes the set of all continuous functions which are strictly increasing and vanishing at zero; $K_{\infty}$ function denotes the set of all functions which are class $K$ functions and unbounded, $C^{i}$ stands for a set of functions with continuous $i$ th partial derivatives, $\|\cdot\|$ represents the Euclidean norm.

\section{Problem statement and preliminaries}

Consider the following switched nonlinear system with state and input unmodeled dynamics:

$$
\left\{\begin{array}{l}
\dot{z}=q(z, y) \\
\dot{x}_{1}=x_{2}+f_{1, \sigma(t)}(y)+\Delta_{1, \sigma(t)}(z, y, t), \\
\dot{x}_{2}=x_{3}+f_{2, \sigma(t)}(y)+\Delta_{2, \sigma(t)}(z, y, t), \\
\vdots \\
\dot{x}_{\rho}=x_{\rho+1}+f_{\rho, \sigma(t)}(y)+\Delta_{\rho, \sigma(t)}(z, y, t)+b_{m, \sigma(t)} v, \\
\vdots \\
\dot{x}_{n-1}=x_{n}+f_{n-1, \sigma(t)}(y)+\Delta_{n-1, \sigma(t)}(z, y, t)+b_{1, \sigma(t)} v \\
\dot{x}_{n}=f_{n, \sigma(t)}(y)+\Delta_{n, \sigma(t)}(z, y, t)+b_{0, \sigma(t)} v \\
y=x_{1}
\end{array}\right.
$$

The minimal realization of input ummodeled dynamics is represented as

$$
\left\{\begin{array}{l}
\dot{\xi}=A_{\Delta, \sigma(t)}(\xi)+b_{\Delta, \sigma(t)} u \\
v=C_{\Delta, \sigma(t)}(\xi)+d_{\Delta, \sigma(t)} u
\end{array}\right.
$$

where $x=\left[x_{1}, x_{2}, \cdots, x_{n}\right]^{T} \in R^{n}$ and $u \in R$ are the unmeasured system states and input, respectively. $y \in R$ is the measured system output; $\sigma(t):[0,+\infty) \rightarrow M=\{1,2, \cdots, m\}$ is the switching signal and all system states do not jump at each switching instant, $t_{0}=0$; For any $i=1,2, \cdots, n, k=1,2, \cdots, m, f_{i, k}(\cdot)$ is the unknown smooth nonlinear function; $z \in R^{n_{0}}$ is the state unmodeled dynamics; $\Delta_{i, k}(z, y, t), i=1,2, \cdots, n$, is the external dynamic disturbance, which is an unknown smooth nonlinear function; $q(z, y)$ is the unknown continuous function; $v \in R$ is the unmeasured signal which acts upon the nonlinear system; $\xi \neq 0 \in R^{q}$ is the input unmodeled dynamic; $A_{\Delta, k}(\cdot), b_{\Delta, k}$ are unknown vectors; $C_{\Delta, k}(\cdot)$ is an unknown function and $d_{\Delta, k}$ is an unknown constant. $b_{i, k} \neq 0, i=1,2, \cdots, n$, is the unknown control coefficient; $\rho+m=n$.

Remark 1. It should be emphasized that switched system (1),(2) does not have strict feedback or pure feedback structures studied in [10, 15-18]. Furthermore, due to the existence of the state and input unmodeled dynamics, finite-time tracking control for switched system (1),(2) becomes more difficult. 
The objective is to design a controller and adaptive laws for switched system $(1),(2)$ such that the output $y$ follows the specified desired trajectory $y_{r}$, and the tracking error can converge to a small neighbourhood of zero in finite time under arbitrary switchings.

Assumption 1 [29-31]. The external disturbance $\Delta_{i, k}(z, y, t), i=1,2, \cdots, n$, is an unknown smooth function satisfying

$$
\left|\Delta_{i, k}(z, y, t)\right| \leq \phi_{i 1, k}(\|z\|)+\phi_{i 2, k}(\|y\|)
$$

where $\phi_{i 1, k}(\cdot) \geq 0$ is an unknown increasing function and $\phi_{i 2, k}(\cdot) \geq 0$ is an unknown smooth function.

Assumption 2 [33-35]. The input ummodeled dynamics (2) has relative degree zero, that is, $d_{\Delta, k} \neq 0$, and there exists an unknown positive constant $\bar{C}_{k}>0$ such that $\left|C_{\Delta, k}(\xi(t))\right| \leq$ $\bar{C}_{k}\|\xi(t)\|$.

Assumption 3 [29-31]. The system $\dot{z}=q(z, y)$ is exponentially input-state-practically stable (exp-ISPS), that is, there exists a $C^{1}$ function $V_{0}(z)$ such that

$$
\begin{gathered}
\bar{\alpha}_{1}(\|z\|) \leq V_{0}(z) \leq \bar{\alpha}_{2}(\|z\|), \\
\frac{\partial V_{0}(z)}{\partial z} q(z, y) \leq-c V_{0}(z)+\gamma(\|y\|)+d,
\end{gathered}
$$

where $\bar{\alpha}_{1}(\cdot), \bar{\alpha}_{2}(\cdot), \gamma(\cdot)$ are the class $K_{\infty}$ functions, $c>0, d \geq 0$ are constants.

Assumption 4 [33-35]. For input unmodeled dynamics (2), there exists a $C^{1}$ function $\bar{V}(\xi)$ satisfying

$$
\begin{gathered}
\beta_{1}\|\xi\|^{2} \leq \bar{V}(\xi) \leq \beta_{2}\|\xi\|^{2}, \\
\frac{\partial \bar{V}(\xi)}{\partial \xi} A_{\Delta, k}(\xi) \leq-2 \delta_{0, k} \bar{V}(\xi), \\
\left\|\frac{\partial \bar{V}(\xi)}{\partial \xi}\right\| \leq \beta_{3}\|\xi\|,
\end{gathered}
$$

where $\beta_{1}>0, \beta_{2}>0, \beta_{3}>0$ and $\delta_{0, k}>0$ are constants.

Assumption 5. The desired trajectory $x_{r}=\left[y_{r}, \dot{y}_{r}\right]^{T} \in \Omega_{r}$ is known, where $\Omega_{r}=\left\{x_{r}\right.$ : $\left.y_{r}^{2}+\dot{y}_{r}^{2} \leq D_{0}\right\}$, and $D_{0}$ is a constant.

Remark 2. In [10, 29-31, 33-35], the upper bounds of control coefficients should be known, and the second derivative of tracking signals was required to be bounded, which are somewhat strict. In this paper, we relax these restrictions, and do not require any information about the second derivative of tracking signals.

Lemma 1 [26]. If $V_{0}$ is a $C^{1}$ function for a system $\dot{z}=q(z, y)$ such that (3) and (4) hold, then, for any constant $\bar{c}^{*} \in(0, c)$, any initial instant $t_{0} \geq 0$, any initial condition 
$z_{0}=z\left(t_{0}\right), \gamma_{0}>0$, any continuous function $\bar{\gamma}(\|y\|)$ satisfying $\bar{\gamma}(\|y\|) \geq \gamma(\|y\|)$, there exist a finite constant $T_{0}=\max \left\{0, \ln \left(V_{0}(z) / \gamma_{0}\right) /\left(c-\bar{c}^{*}\right)\right\} \geq 0$, a function $D\left(t_{0}, t\right) \geq 0$ and an unmeasured dynamic signal described by

$$
\dot{v}=-\bar{c}^{*} v+\bar{\gamma}(\|y\|)+d, v\left(t_{0}\right)=v_{0}, v_{0} \geq 0,
$$

such that $V_{0}(z) \leq v(t)+D\left(t, t_{0}\right)$, where $D\left(t, t_{0}\right)=\max \left\{0, e^{-c\left(t-t_{0}\right)} V_{0}(z)-e^{-\bar{c}^{*}\left(t-t_{0}\right)} \gamma_{0}\right\}$, $D\left(t, t_{0}\right)=0$ for $t \geq T_{0}+t_{0}$. Without loss of generality, we assume $\bar{\gamma}(\|y\|)=\gamma(\|y\|)$.

Lemma 2 [26]. For any continuous function $f(x, y)$, there exist smooth functions $\varphi_{1}(x) \geq$ $0, \varphi_{2}(y) \geq 0$, such that the following inequality holds:

$$
|f(x, y)| \leq \varphi_{1}(x)+\varphi_{2}(y), \forall x, y \in R .
$$

Lemma 3 [40]. Consider a switched nonlinear system $\dot{x}=f(x)$, if there exist a positive definite function $V: R^{n} \rightarrow R$, and constants $0<\alpha<1, c>0, d \geq 0$, such that, for any $x_{0} \in \Omega_{0} \subset R^{n}$, the following inequality holds:

$$
\dot{V}(x) \leq-c V^{\alpha}(x)+d,
$$

then,

$$
V^{\alpha}(x) \leq \frac{d}{c(1-\eta)}, \forall t \geq T^{*}=\frac{1}{c \eta(1-\alpha)}\left(V\left(x\left(t_{0}\right)\right)\right)^{1-\alpha}+t_{0},
$$

where $0<\eta<1$, and $t_{0}$ is the initial time.

Lemma 4 [43]. For any unknown continuous function $h(z)$, a neural network can be constructed as the following form to approximate it:

$$
h(z)=W^{* T} T(z)+D(z), \forall z \in \Omega_{z},
$$

where $T(z)=\left[T_{1}(z), T_{2}(z), \cdots, T_{l}(z)\right]^{T} \in R^{l}$ is the basic function vector with the node number $l \geq 1, W^{*}=\left[W_{1}^{*}, W_{2}^{*} \cdots, W_{l}^{*}\right]^{T} \in R^{l}$ is the ideal weight vector, and $D(z)$ is the approximate error. The basic function $T_{i}(z)$ is taken as the Gaussian function, which has the following form

$$
T_{i}(z)=\exp \left(-\frac{\left(z-c_{i}\right)^{T}\left(z-c_{i}\right)}{\mu_{i}^{2}}\right), i=1,2, \cdots, l,
$$

where $c_{i}$ is the center of the radial basic function and $\mu_{i}>0$ is the width of the Gaussian function. The value of the ideal weight vector $W^{*}$ is determined by $W$ that minimize the approximate error $D(z)$ for all $z \in \Omega_{z}$ :

$$
W^{*}=\arg \min _{W \in R^{l}}\left\{\sup _{z \in \Omega_{z}}\left|h(z)-W^{T} T(z)\right|\right\} .
$$


Proposition 1 [44]. Let $x \in R, y \in R$ and given any real numbers $c>0, d>0, \gamma>0$, the following inequality holds:

$$
|x|^{c}|y|^{d} \leq \frac{c}{c+d} \gamma|x|^{c+d}+\frac{d}{c+d} \gamma^{-\frac{c}{d}}|y|^{c+d}
$$

Proposition 2. Consider the input unmodeled dynamics satisfying Assumption 4 and the following first-order filter

$$
\dot{\bar{m}}=-\delta_{0} \bar{m}+|u|
$$

the following inequality holds:

$$
\|\xi(t)\| \leq c_{1}^{*}(\|\xi(0)\|+|\bar{m}(0)|) e^{-\delta_{0} t}+c_{2}^{*}|\bar{m}(t)|,
$$

where $\delta_{0}=\min _{k \in M}\left\{\delta_{0, k}\right\}, c_{1}^{*}=\frac{1}{\sqrt{\beta_{1}}} \max \left\{\sqrt{\beta_{2}}, \beta_{4}\right\}, c_{2}^{*}=\frac{\beta_{4}}{\sqrt{\beta_{1}}}$, and $\beta_{4}=\frac{\beta_{3}}{2 \sqrt{\beta_{1}}} \max _{k \in M}\left\{\left\|b_{\Delta, k}\right\|\right\}$.

Proof. According to Assumption 4, we obtain

$$
\dot{\bar{V}}(\xi) \leq \frac{\partial \bar{V}(\xi)}{\partial \xi}\left[A_{\Delta, \sigma(t)}(\xi)+b_{\Delta, \sigma(t)} u\right] \leq-2 \delta_{0, \sigma(t)} \bar{V}(\xi)+\beta_{3}\|\xi\|\left\|b_{\Delta, \sigma(t)}\right\||u| .
$$

Setting $W(\xi)=\sqrt{\bar{V}(\xi)}$, we have

$$
\dot{W}(\xi)=\frac{1}{2 \sqrt{\bar{V}(\xi)}} \dot{\bar{V}}(\xi) \leq-\delta_{0, \sigma(t)} \frac{\bar{V}(\xi)}{\sqrt{\bar{V}(\xi)}}+\frac{\beta_{3}\left\|b_{\Delta, \sigma(t)}\right\|\|\xi\|}{2 \sqrt{\beta_{1}}\|\xi\|}|u| .
$$

From (8), we obtain

$$
W(\xi) \leq W(\xi(0)) e^{-\delta_{0} t}+\beta_{4} \int_{0}^{t} e^{-\delta_{0}(t-\tau)}|u(\tau)| d \tau .
$$

Since $e^{-\delta_{0}(t-\tau)}\left(\dot{\bar{m}}(\tau)+\delta_{0} \bar{m}(\tau)\right)=\frac{d}{d \tau}\left[e^{-\delta_{0}(t-\tau)} \bar{m}(\tau)\right]$, we obtain

$$
\int_{0}^{t} e^{-\delta_{0}(t-\tau)}|u(\tau)| d \tau=\bar{m}(t)-\bar{m}(0) e^{-\delta_{0} t}
$$

According to Assumption 4 and substituting (10) into (9) yields

$$
\|\xi(t)\| \leq\left[\frac{\sqrt{\beta_{2}}}{\sqrt{\beta_{1}}}\|\xi(0)\|+\frac{\beta_{4}}{\sqrt{\beta_{1}}}|\bar{m}(0)|\right] e^{-\delta_{0} t}+\frac{\beta_{4}}{\sqrt{\beta_{1}}} \bar{m}(t) .
$$

Then, (11) can be rewritten as the following form

$$
\|\xi(t)\| \leq c_{1}^{*}(\|\xi(0)\|+|\bar{m}(0)|) e^{-\delta_{0} t}+c_{2}^{*}|\bar{m}(t)| .
$$


The proof of Proposition 2 is completed.

Remark 3. In Proposition 2, the first-order filter (7) plays a critical role in dealing with input unmodeled dynamics. With the aid of the filter (7), the state of the input unmodeled dynamics can be restricted by (12), which will be used for controller design in the later section.

Proposition 3[44]. Let $x \in R, y \in R$, and $p \geq 1$ be a constant, the following inequality holds:

$$
(|x|+|y|)^{1 / p} \leq|x|^{1 / p}+|y|^{1 / p} \leq 2^{(p-1) / p}(|x|+|y|)^{1 / p} .
$$

\section{System parameterization and K-filters design}

In this section, the parameterization of switched system (1) and (2) is given to construct the state observer.

Substituting (2) into (1), we have

$$
\left\{\begin{array}{l}
\dot{z}=q(z, y) \\
\dot{x}_{1}=x_{2}+f_{1, \sigma(t)}(y)+\Delta_{1, \sigma(t)}(z, y, t) \\
\vdots \\
\dot{x}_{\rho}=x_{\rho+1}+f_{\rho, \sigma(t)}(y)+\Delta_{\rho, \sigma(t)}(z, y, t)+b_{m, \sigma(t)} C_{\Delta, \sigma(t)}+b_{m, \sigma(t)} d_{\Delta, \sigma(t)} u \\
\vdots \\
\dot{x}_{n-1}=x_{n}+f_{n-1, \sigma(t)}(y)+\Delta_{n-1, \sigma(t)}(z, y, t)+b_{1, \sigma(t)} C_{\Delta, \sigma(t)}+b_{1, \sigma(t)} d_{\Delta, \sigma(t)} u, \\
\dot{x}_{n}=f_{n, \sigma(t)}(y)+\Delta_{n, \sigma(t)}(z, y, t)+b_{0, \sigma(t)} C_{\Delta, \sigma(t)}+b_{0, \sigma(t)} d_{\Delta, \sigma(t)} u \\
y=x_{1}
\end{array}\right.
$$

In order to facilitate the parameterization, we let

$$
A=\left[\begin{array}{ccccc}
0 & 1 & 0 & \cdots & 0 \\
0 & 0 & 1 & \cdots & 0 \\
\vdots & \vdots & \vdots & \ddots & \vdots \\
0 & 0 & 0 & \cdots & 0
\end{array}\right], F_{\sigma(t)}=\left[\begin{array}{c}
f_{1, \sigma(y)}(y) \\
f_{2, \sigma(y)}(y) \\
\vdots \\
f_{n, \sigma(y)}(y)
\end{array}\right], \Delta_{\sigma(t)}=\left[\begin{array}{c}
\Delta_{1, \sigma(t)} \\
\Delta_{2, \sigma(t)} \\
\vdots \\
\Delta_{n, \sigma(t)}
\end{array}\right] .
$$

Then, (13) can be rewritten as follows

$$
\left\{\begin{aligned}
\dot{z} & =q(z, y) \\
\dot{x} & =A x+F_{\sigma(t)}(y)+\Delta_{\sigma(t)}(z, y, t)+\sum_{r=0}^{m} e_{n-r} b_{r, \sigma(t)} C_{\Delta, \sigma(t)}+\sum_{r=0}^{m} e_{n-r} b_{r, \sigma(t)} d_{\Delta, \sigma(t)} u \\
y & =e_{1}^{T} x .
\end{aligned}\right.
$$

Since $f_{i, k}(y)$ is a continuous function, we adopt radial basic function neural networks $\hat{f}_{i, k}(y)=$ $\theta_{i, k}^{T} G_{i}(y)$ to approximate it on the compact set $y \in \Omega_{y} \subset R, \theta_{i, k}=\left[\theta_{i 1, k}, \cdots, \theta_{i N_{i}, k}\right]^{T} \in R^{N_{i}}$ represents the weight vector, $N_{i}>1$ is the number of neuron nodes, $G_{i}(y)=\left[G_{i 1}(y), G_{i 2}(y)\right.$, 
$\left.\cdots, G_{i N_{i}}(y)\right]^{T} \in R^{N_{i}}$ is the basic function vector and it is chosen as the commonly used Gaussian function with the following form

$$
G_{i j}(y)=\exp \left(-\frac{\left(y-\kappa_{i j}\right)^{2}}{b_{i j}^{2}}\right), i=1,2, \cdots, n, j=1,2, \cdots, N_{i},
$$

where $\kappa_{i j}$ is the center of the receptive field, $b_{i j}>0$ is the width of the Gaussian function. $\theta_{i, k}^{*}$ represents the unknown ideal weight vector defined as follows

$$
\theta_{i, k}^{*}=\arg \min _{\theta_{i, k} \in R^{N_{i}}}\left\{\sup _{y \in \Omega_{y}}\left|\theta_{i, k}^{T} G_{i}(y)-f_{i, k}(y)\right|\right\},
$$

then, we have

$$
f_{i, \sigma(t)}(y)=\theta_{i, \sigma(t)}^{* T} G_{i}(y)+\delta_{i, \sigma(t)}(y)
$$

where $\delta_{i, \sigma(t)}(y)$ denotes the approximation error.

In view of (15), (14) can be represented as the following form

$$
\left\{\begin{aligned}
\dot{z} & =q(z, y) \\
\dot{x} & =A x+S^{T}(y) \theta_{h, \sigma(t)}+\delta_{\sigma(t)}+\Delta_{\sigma(t)}(z, y, t)+\sum_{r=0}^{m} e_{n-r} b_{r, \sigma(t)} C_{\Delta, \sigma(t)} \\
& +\sum_{r=0}^{m} e_{n-r} b_{r, \sigma(t)} d_{\Delta, \sigma(t)} u \\
y & =e_{1}^{T} x
\end{aligned}\right.
$$

where

$$
G^{T}(y)=\left[\begin{array}{cccc}
G_{1}^{T}(y) & & & \\
& G_{2}^{T}(y) & & \\
& & \ddots & \\
& & & G_{n}^{T}(y)
\end{array}\right], \theta_{h, \sigma(t)}^{*}=\left[\begin{array}{c}
\theta_{1, \sigma(t)}^{*} \\
\theta_{2, \sigma(t)}^{*} \\
\vdots \\
\theta_{n, \sigma(t)}^{*}
\end{array}\right], \delta_{\sigma(t)}=\left[\begin{array}{c}
\delta_{1, \sigma(t)}(y) \\
\delta_{2, \sigma(t)}(y) \\
\vdots \\
\delta_{n, \sigma(t)}(y)
\end{array}\right]
$$

Further, (16) can be expressed as follows

$$
\left\{\begin{array}{l}
\dot{z}=q(z, y) \\
\dot{x}=A x+F^{T}(y, u) \theta_{1, \sigma(t)}+\delta_{\sigma(t)}+\Delta_{\sigma(t)}(z, y, t)+\sum_{r=0}^{m} e_{n-r} b_{r, \sigma(t)} C_{\Delta, \sigma(t)}, \\
y=e_{1}^{T} x
\end{array}\right.
$$

where

$$
F^{T}(y, u)=\left[\left[\begin{array}{c}
0_{(\rho-1) \times(m+1)} \\
I_{m+1}
\end{array}\right] u, G^{T}(y)\right], \theta_{1, \sigma(t)}=\left[\begin{array}{c}
b_{m, \sigma(t)} d_{\Delta, \sigma(t)} \\
\vdots \\
b_{1, \sigma(t)} d_{\Delta, \sigma(t)} \\
b_{0, \sigma(t)} d_{\Delta, \sigma(t)} \\
\theta_{h, \sigma(t)}^{*}
\end{array}\right] .
$$


Since the states of system (1) are unavailable, the following filters are employed to reconstruct the states

$$
\left\{\begin{array}{l}
\dot{\xi}_{0}=A_{0} \xi_{0}+L y, \xi_{0} \in R^{n}, \\
\dot{\Omega}^{T}=A_{0} \Omega^{T}+F^{T}(y, u), \Omega^{T} \in R^{n \times\left((m+1)+\sum_{i=1}^{n} N_{i}\right)}
\end{array}\right.
$$

where $A_{0}=A-L C^{T}=\left[\begin{array}{cccc}-l_{1} & & \\ -l_{2} & & I_{n-1} & \\ \vdots & & & \\ -l_{n} & 0 & \cdots & 0\end{array}\right], L=\left[\begin{array}{c}l_{1} \\ l_{2} \\ \vdots \\ l_{n}\end{array}\right],\left|s I-A_{0}\right|=s^{n}+l_{1} s^{n-1}+\cdots+$ $l_{n-1} s+l_{n}$ is a Hurwitz polynomial, $A_{0}^{T} P+P A_{0}=-h I, h>0$ is a design constant, $P=$ $P^{T}>0$.

Denote the first $(m+1)$ columns of $\Omega^{T}$ by $\mu_{m}, \cdots, \mu_{1}, \mu_{0}$, then, we get $\Omega^{T}=\left[\mu_{m}, \cdots, \mu_{1}, \mu_{0}, \Xi\right]$. According to (18), the vectors $\mu_{m}, \cdots, \mu_{1}, \mu_{0}$ are generated by only one input filter

$$
\dot{\mu}_{r}=A_{0} \mu_{r}+e_{n-r} u, r=0,1, \cdots, m, u_{r} \in R^{n \times(m+1)} .
$$

It is easy to show that $A_{0}^{r} e_{n}=e_{n-r}, r=0,1, \cdots, m$. Let

$$
\mu_{r}=A_{0}^{r} \lambda, r=0,1, \cdots, m, \mu_{r} \in R^{n \times(m+1)} .
$$

Then, (19) can be rewritten as the following form

$$
\dot{\lambda}=A_{0} \lambda+e_{n} u
$$

Let $\mu_{r, i}, r=0,1, \cdots, m, i=1,2, \cdots, n$, be the $i$ th element of the vector $\mu_{r}$, and $\lambda_{k}$ be the $k$ th element of the vector $\lambda$, respectively. Based on the discussion of [26], we have

$$
\mu_{r, i}=[*, *, \cdots, 1]\left[\begin{array}{c}
\lambda_{1} \\
\lambda_{2} \\
\vdots \\
\lambda_{r+i}
\end{array}\right], \lambda_{k}=0, k>n
$$

where $*$ is the polynomial consisting of $l_{1}, l_{2}, \cdots, l_{n}$. Since $l_{1}, l_{2}, \cdots, l_{n}$ are design parameters, the polynomial which $*$ represents is bounded.

According to (18)-(20), K-filters used for state estimation can be described as follows

$$
\left\{\begin{array}{l}
\dot{\xi}_{0}=A_{0} \xi_{0}+L y, \xi_{0} \in R^{n}, \\
\dot{\lambda}=A_{0} \lambda+e_{n} u, \lambda \in R^{n}, \\
\dot{\Xi}=A_{0} \Xi+G^{T}(y), \Xi \in R^{n \times \sum_{i=1}^{n} N_{i}} .
\end{array}\right.
$$


The nominal state estimate is $\hat{x}=\xi_{0}+\Omega^{T} \theta_{1, \sigma(t)}$ and the observe error is $\varepsilon=x-\hat{x}$. Therefore, the states can be rewritten as $x=\Omega^{T} \theta_{1, \sigma(t)}+\xi_{0}+\varepsilon$, and we have

$$
x=\xi_{0}+\sum_{r=0}^{m} \mu_{r} b_{r, \sigma(t)} d_{\Delta, \sigma(t)}+\Xi \theta_{h, \sigma(t)}^{*}+\varepsilon .
$$

Further, according to (22), the observer error equation can be expressed as

$$
\dot{\varepsilon}=A_{0} \varepsilon+\delta_{\sigma(t)}(y)+\Delta_{\sigma(t)}(z, y, t)+\sum_{r=0}^{m} e_{n-r} b_{r, \sigma(t)} C_{\Delta, \sigma(t)}(\xi) .
$$

\section{Adaptive dynamic surface controller design}

In this section, a tracking control scheme based on DSC technique will be developed for switched system (1),(2), which makes that the tracking error can converge to a small neighbourhood of zero in finite time under arbitrary switchings. The whole design procedure needs $\rho$ steps based on the following coordinate transformations: $S_{1}=y-y_{r}, S_{i}=\mu_{m, i}-w_{i}, i=$ $2,3, \cdots, \rho, w_{i}$ is the output of a first-order filter.

Step 1. Taking the derivative of $S_{1}$, and combining (1) with (23), we have

$$
\begin{aligned}
\dot{S}_{1} & =x_{2}+f_{1, \sigma(t)}(y)+\Delta_{1, \sigma(t)}(z, y, t) \\
& =\mu_{m, 2} b_{m, \sigma(t)} d_{\Delta, \sigma(t)}+\bar{\omega}^{T} \bar{\theta}_{1, \sigma(t)}+\delta_{1, \sigma(t)}(y)+\Delta_{1, \sigma(t)}(z, y, t)+\varepsilon_{2}-\dot{y}_{r},
\end{aligned}
$$

where $\bar{\omega}=\left[\xi_{0,2}, 0, \mu_{m-1,2}, \cdots, \mu_{1,2}, \mu_{0,2}, \Xi_{(2)}+G_{(1)}^{T}(y)\right], \Xi_{(2)}$ and $G_{(1)}^{T}(y)$ denote the second and first rows of the matrices $\Xi$ and $G^{T}(y)$, respectively. $\bar{\theta}_{1, \sigma(t)}=\left[1, \theta_{1, \sigma(t)}^{T}\right]^{T}$.

Choose a Lyapunov function candidate for the first step as follows

$$
\begin{gathered}
V_{\varepsilon}=\frac{B_{0}^{L}}{\gamma_{\varepsilon}} \varepsilon^{T} P \varepsilon, \\
V_{S_{1}}=\frac{1}{2} S_{1}^{2}, \\
V_{1}=V_{S_{1}}+\frac{\gamma_{0}}{2} B_{0}^{L} \tilde{\theta}_{0}^{2}+\frac{\gamma_{1}}{2} B_{0}^{L} \tilde{\theta}_{1}^{2}+\frac{\gamma_{H}}{2} B_{0}^{L} \tilde{H}^{2}+\frac{v}{\lambda^{*}}+V_{\varepsilon},
\end{gathered}
$$

where $\tilde{\theta}_{0}=\theta_{0}-\hat{\theta}_{0}, \tilde{\theta}_{1}=\theta_{1}-\hat{\theta}_{1}, \tilde{H}=H-\hat{H}, \hat{\theta}_{0}$ is the estimate of $\theta_{0}=L_{0}^{(1+\tau) / 2}\left\|W_{0}^{*}\right\|^{1+\tau}$. $L_{0}, \tau, W_{0}^{*}, \theta_{1}, H$ will be given later. $\gamma_{0}>0, \gamma_{1}>0, \gamma_{H}>0, \gamma_{\varepsilon}>0, \lambda^{*}>0$ are design parameters. $B_{0, \sigma(t)}=b_{m, \sigma(t)} d_{\Delta, \sigma(t)}, B_{0}^{L}=\min _{k \in M}\left\{B_{0, k}\right\}$.

Differentiating $V_{S_{1}}$ with respect to time $t$, and combining Assumption 1 with (15), we have

$$
\dot{V}_{S_{1}}=S_{1} \mu_{m, 2} b_{m, \sigma(t)} d_{\Delta, \sigma(t)}+S_{1} \bar{\omega}^{T} \bar{\theta}_{1, \sigma(t)}+S_{1} \delta_{1, \sigma(t)}(y)+S_{1} \Delta_{1, \sigma(t)}(z, y, t)+S_{1} \varepsilon_{2}-S_{1} \dot{y}_{r}
$$


According to Assumption $3, \bar{\alpha}_{1}(\cdot)$ is a class $K_{\infty}$ function, therefore, $\bar{\alpha}_{1}^{-1}(\cdot)$ is a non-decreasing function. From Lemma 1 and Lemma 2, we have

$$
\begin{aligned}
\left|S_{1}\right|\left|\Delta_{1, \sigma(t)}(z, y, t)\right| & \leq\left|S_{1}\right| \phi_{11, \sigma(t)} \circ \bar{\alpha}_{1}^{-1}\left(v(t)+D\left(t, t_{0}\right)\right)+\left|S_{1}\right| \phi_{12, \sigma(t)}(|y|) \\
& \leq\left|S_{1}\right| \varphi_{11, \sigma(t)}(v(t))+\left|S_{1}\right| \varphi_{12, \sigma(t)}\left(D\left(t, t_{0}\right)\right)+\left|S_{1}\right| \phi_{12, \sigma(t)}(|y|)
\end{aligned}
$$

where $\varphi_{11, k}(\cdot) \geq 0$ and $\varphi_{12, k}(\cdot) \geq 0, k=1,2, \cdots, m$, are two unknown smooth functions.

According to Lemma 1 , one can show that $D\left(t, t_{0}\right)$ is a bounded function, thus, there exists a constant $\bar{\theta}_{1, k}^{*}>0, k=1,2, \cdots, m$, satisfying $\varphi_{12, k}\left(D\left(t, t_{0}\right)\right) \leq \bar{\theta}_{1, k}^{*}$. Noting (30), we obtain

$$
\begin{aligned}
\left|S_{1}\right|\left|\Delta_{1, \sigma(t)}(z, y, t)\right| & \leq\left|S_{1}\right| \varphi_{11, \sigma(t)}(v(t))+\left|S_{1}\right| \varphi_{12, \sigma(t)}\left(D\left(t, t_{0}\right)\right)+\left|S_{1}\right| \phi_{12, \sigma(t)}(|y|) \\
& \leq B_{0}^{L} S_{1}^{2} \varphi_{11, \sigma(t)}^{2}+B_{0}^{L} S_{1}^{2} \bar{\theta}_{1, \sigma(t)}^{* 2}+B_{0}^{L} S_{1}^{2} \phi_{12, \sigma(t)}^{2}(|y|)+\frac{3}{4 B_{0}^{L}}
\end{aligned}
$$

Furthermore, in view of (29), and using the inequality as shown in Proposition 1, we have

$$
S_{1} \bar{\omega}^{T} \bar{\theta}_{1, \sigma(t)} \leq\left|S_{1}\right|\|\bar{\omega}\|\left\|\bar{\theta}_{1, \sigma(t)}\right\| \leq \frac{\tau}{(1+\tau) a_{11}^{\tau(1+\tau)}\left(B_{0}^{L}\right)^{1 / \tau}}+\frac{a_{11}^{\tau(1+\tau)}}{1+\tau}\left|S_{1}\right|^{1+\tau} \bar{\omega}^{*} \theta_{1} B_{0}^{L},
$$

where $\tau \in R_{\text {ood }}^{+}$is a constant satisfying $0<\tau<1 . \bar{\omega}^{*}=\|\bar{\omega}\|^{1+\tau}$ and $\theta_{1}=\left\{\max _{k \in M}\left\{\left\|\bar{\theta}_{1, k}\right\|\right\}\right\}^{1+\tau}$ are two unknown positive constants. $a_{11}>0$ is a design parameter.

Remark 4. According to (32), we just need to estimate the scalar $\theta_{1}$ rather than the vector $\bar{\theta}_{1, k}$, which makes that the number of adaptive parameters is greatly reduced and the burdensome computation is alleviated.

Substituting (31) and (32) into (29), we obtain

$$
\begin{aligned}
\dot{V}_{S_{1}} \leq & S_{1} \mu_{m, 2} b_{m, \sigma(t)} d_{\Delta, \sigma(t)}+\frac{a_{11}^{\tau(1+\tau)}}{1+\tau}\left|S_{1}\right|^{1+\tau} \bar{\omega}^{*} \theta_{1} B_{0}^{L}+B_{0}^{L} S_{1}^{2} \varphi_{11, \sigma(t)}^{2}+B_{0}^{L} S_{1}^{2} \bar{\theta}_{1, \sigma(t)}^{* 2} \\
& +B_{0}^{L} S_{1}^{2} \dot{y}_{r}^{2}+B_{0}^{L} S_{1}^{2} \phi_{12, \sigma(t)}^{2}(|y|)+B_{0}^{L} S_{1}^{2} \delta_{1, \sigma(t)}^{2}(y)+B_{0}^{L} \varepsilon^{T} \varepsilon+\frac{5}{4 B_{0}^{L}}+\frac{1}{B_{0}^{L}} S_{1}^{2} \\
& +\frac{\tau}{(1+\tau) a_{11}^{\tau(1+\tau)}\left(B_{0}^{L}\right)^{1 / \tau}} .
\end{aligned}
$$

Taking the derivative of $V_{\varepsilon}$, and combining Assumption 1, Assumption 2 with Lemma 2, we have

$$
\begin{aligned}
\dot{V}_{\varepsilon}= & \frac{B_{0}^{L}}{\gamma_{\varepsilon}}\left[\varepsilon^{T}\left(A_{0}^{T} P+P A_{0}\right) \varepsilon\right]+\frac{2 B_{0}^{L}}{\gamma_{\varepsilon}} \varepsilon^{T} P \delta_{\sigma(t)}(y)+\frac{2 B_{0}^{L}}{\gamma_{\varepsilon}} \varepsilon^{T} P \Delta_{\sigma(t)}+\frac{B_{0}^{L}}{\gamma_{\varepsilon}} \varepsilon^{T} P B_{\sigma(t)} C_{\Delta, \sigma(t)} \\
\leq & -\frac{B_{0}^{L}}{\gamma_{\varepsilon}}(h-3) \varepsilon^{T} \varepsilon+\frac{B_{0}^{L}}{\gamma_{\varepsilon}}\|P\|^{2}\left\|\delta_{\sigma(t)}(y)\right\|^{2}+\frac{2 B_{0}^{L}}{\gamma_{\varepsilon}}\|P\|^{2} \phi_{0, \sigma(t)}(v(t))+\frac{2 B_{0}^{L}}{\gamma_{\varepsilon}}\|P\|^{2} \theta_{0, \sigma(t)}^{*} \\
& +\frac{2 B_{0}^{L}}{\gamma_{\varepsilon}}\|P\|^{2} \sum_{i=1}^{n} \phi_{i 2, \sigma(t)}^{2}(|y|)+\frac{2 B_{0}^{L}}{\gamma_{\varepsilon}}\|P\|^{2}\left\|B_{\sigma(t)} C_{\Delta, \sigma(t)}(\xi)\right\|^{2},
\end{aligned}
$$


where $\phi_{0, k}(\cdot) \geq 0$ is an unknown smooth function and $\theta_{0, k}^{*} \geq 0$ is an unknown constant. Further, using Proposition 2, we obtain

$$
\begin{aligned}
\frac{\left|C_{\Delta, \sigma(t)}(\xi(t))\right|}{1+|\bar{m}(t)|} & \leq \frac{\bar{C}_{\sigma(t)} c_{1}^{*}(\|\xi(0)\|+|\bar{m}(0)|) e^{-\delta_{0} t}+\bar{C}_{\sigma(t)} c_{2}^{*}|\bar{m}(t)|}{1+|\bar{m}(t)|} \\
& \leq \max \left\{\bar{C}_{\sigma(t)} c_{1}^{*}(\|\xi(0)\|+|\bar{m}(0)|), \bar{C}_{\sigma(t)} c_{2}^{*}\right\}=H_{m, \sigma(t)},
\end{aligned}
$$

where $H_{m, k}>0$ is an unknown constant.

Let $H_{\sigma(t)}=\|P\|^{2}\left\|B_{\sigma(t)}\right\|^{2} H_{m, \sigma(t)}^{2}, H=\left\{\max _{k \in M}\left\{H_{k}\right\}\right\}^{1+\tau}$, then we have

$$
\|P\|^{2}\left\|B_{\sigma(t)}\right\|^{2}\left|C_{\Delta, \sigma(t)}(\xi)\right|^{2}=(1+|\bar{m}|)^{2}\|P\|^{2}\left\|B_{\sigma(t)}\right\|^{2}\left(\frac{\left|C_{\Delta, \sigma(t)}(\xi)\right|}{1+|\bar{m}|}\right)^{2} \leq\left(P_{m} H\right)^{1 /(1+\tau)},
$$

where $P_{m}=(1+|\bar{m}|)^{2(1+\tau)}$.

Taking the derivative of $V_{1}$, and substituting (33)-(36) into it leads to

$$
\begin{aligned}
\dot{V}_{1} \leq & S_{1} \mu_{m, 2} b_{m, \sigma(t)} d_{\Delta, \sigma(t)}+\frac{a_{11}^{\tau(1+\tau)}}{1+\tau}\left|S_{1}\right|^{1+\tau} \bar{\omega}^{*} \theta_{1} B_{0}^{L}+B_{0}^{L} S_{1}^{2} \varphi_{11, \sigma(t)}^{2}+B_{0}^{L} S_{1}^{2} \bar{\theta}_{1, \sigma(t)}^{* 2}+\frac{5}{4 B_{0}^{L}} \\
& +\frac{1}{B_{0}^{L}} S_{1}^{2}+B_{0}^{L} \varepsilon^{T} \varepsilon-\frac{B_{0}^{L}}{\gamma_{\varepsilon}}(h-3) \varepsilon^{T} \varepsilon+\frac{B_{0}^{L}}{\gamma_{\varepsilon}}\|P\|^{2}\left\|\delta_{\sigma(t)}(y)\right\|^{2}+\frac{2 B_{0}^{L}}{\gamma_{\varepsilon}}\|P\|^{2} \phi_{0, \sigma(t)}(v(t)) \\
& +\frac{2 B_{0}^{L}}{\gamma_{\varepsilon}}\|P\|^{2} \theta_{0, \sigma(t)}^{*}+\frac{2 B_{0}^{L}}{\gamma_{\varepsilon}}\|P\|^{2} \sum_{i=1}^{n} \phi_{i 2, \sigma(t)}^{2}(|y|)+\frac{2 B_{0}^{L}}{\gamma_{\varepsilon}}\left(P_{m} H\right)^{1 /(1+\tau)}-\gamma_{0} B_{0}^{L} \tilde{\theta}_{0} \dot{\hat{\theta}}_{0} \\
& -\gamma_{1} B_{0}^{L} \tilde{\theta}_{1} \dot{\hat{\theta}}_{1}-\gamma_{H} B_{0}^{L} \tilde{H} \dot{\hat{H}}-\frac{\bar{c}^{*}}{\lambda^{*}} v+\frac{\gamma(\|y\|)}{\lambda^{*}}+\frac{d}{\lambda^{*}}+\frac{\tau}{(1+\tau) a_{11}^{\tau(1+\tau)}\left(B_{0}^{L}\right)^{1 / \tau}} \\
& +B_{0}^{L} S_{1}^{2} \dot{y}_{r}^{2}+B_{0}^{L} S_{1}^{2} \phi_{12, \sigma(t)}^{2}(|y|)+B_{0}^{L} S_{1}^{2} \delta_{1, \sigma(t)}^{2}(y) .
\end{aligned}
$$

Taking the virtual control law for the first step as follows

$\alpha_{1}=-S_{1}^{\tau}\left(\frac{a_{11}^{\tau(1+\tau)}}{1+\tau} \bar{\omega}^{*} \sqrt{1+\hat{\theta}_{1}^{2}}+\frac{a_{12}^{\tau(1+\tau)}}{1+\tau} \sqrt{1+\hat{\theta}_{0}^{2}}+\frac{1}{1+\tau}+\frac{2 S_{1}^{1+\tau} a_{13}^{\tau(1+\tau)} P_{m}}{\gamma_{\varepsilon} \varepsilon^{* 2}(1+\tau)} \sqrt{1+\hat{H}^{2}}\right)-c_{1} S_{1}$,

where $c_{1}>0, a_{12}>0, a_{13}>0$ and $\varepsilon^{*}>0$ are four design parameters.

A first-order filter is designed as follows

$$
\tau_{2} \dot{w}_{2}+w_{2}=\alpha_{1}, w_{2}(0)=\alpha_{1}(0)
$$

where $\tau_{2}>0$ is a constant. Let $y_{2}=w_{2}-\alpha_{1}$, then $\dot{w}_{2}=-\frac{y_{2}}{\tau_{2}}$. 
Since $\mu_{m, 2}=y_{2}+\alpha_{1}+S_{2}$, we obtain from (37) that

$$
\begin{aligned}
\dot{V}_{1} \leq & \frac{1}{2} S_{2}^{2}+S_{1} \alpha_{1} B_{0, \sigma(t)}+\frac{a_{11}^{\tau(1+\tau)}}{1+\tau}\left|S_{1}\right|^{1+\tau} \bar{\omega}^{*} \theta_{1} B_{0}^{L}+B_{0}^{L}\left|S_{1}\right| \phi_{0}\left(X_{1}\right)+\frac{1}{2} y_{2}^{2}+\frac{5}{4 B_{0}^{L}} \\
& +\frac{2 B_{0}^{L} a_{13}^{\tau(1+\tau)} P_{m} H S_{1}^{2(1+\tau)}}{\gamma_{\varepsilon} \varepsilon^{* 2}(1+\tau)}+\left(1-\frac{S_{1}^{2}}{\varepsilon^{* 2}}\right) \frac{2 B_{0}^{L}}{\gamma_{\varepsilon}}\left(P_{m} H\right)^{1 /(1+\tau)}-\frac{B_{0}^{L}}{\gamma_{\varepsilon}}\left(h-3-\gamma_{\varepsilon}\right) \varepsilon^{T} \varepsilon \\
& -\gamma_{0} B_{0}^{L} \tilde{\theta}_{0} \dot{\hat{\theta}}_{0}-\gamma_{1} B_{0}^{L} \tilde{\theta}_{1} \dot{\hat{\theta}}_{1}-\gamma_{H} B_{0}^{L} \tilde{H} \dot{\hat{H}}-\frac{\bar{c}^{*}}{\lambda^{*}} v+\frac{d}{(1+\tau) a_{11}^{\tau(1+\tau)}\left(B_{0}^{L}\right)^{1 / \tau}}+\frac{d}{\lambda^{*}} \\
& +\frac{2 \tau B_{0}^{L}}{\varepsilon^{* 2} \gamma_{\varepsilon}(1+\tau) a_{13}^{\tau(1+\tau)}}+\left(1-\frac{S_{1}^{2}}{\varepsilon^{* 2}}\right) Q(y, v) B_{0}^{L},
\end{aligned}
$$

where $\phi_{0}\left(X_{1}\right)=\max _{k \in M}\left\{\left|S_{1}\right|\left(\varphi_{11, k}^{2}(v(t))+\bar{\theta}_{1, k}^{* 2}+\phi_{12, k}^{2}(|y|)+\frac{1}{\left(B_{0}^{L}\right)^{2}}+\frac{1}{\varepsilon^{* 2}} Q(y, v)+\frac{\left(B_{0}^{M}\right)^{2}}{B_{0}^{L}}+\right.\right.$ $\left.\left.\dot{y}_{r}^{2}+\delta_{1, \sigma(t)}^{2}(y)\right)\right\}, X_{1}=\left[y, v, y_{r}, \dot{y}_{r}\right]^{T}, Q(y, v)=\max _{k \in M}\left\{\frac{1}{\gamma_{\varepsilon}}\|P\|^{2}\left\|\delta_{k}(y)\right\|^{2}+\frac{2}{\gamma_{\varepsilon}}\|P\|^{2} \phi_{0, k}(v(t))+\right.$ $\left.\frac{2}{\gamma_{\varepsilon}}\|P\|^{2} \theta_{0, k}^{*}+\frac{2}{\gamma_{\varepsilon}}\|P\|^{2} \sum_{i=1}^{n} \phi_{i 2, k}^{2}(|y|)+\frac{\gamma(\|y\|)}{\lambda^{*}}\right\}, B_{0}^{M}=\max _{k \in M}\left\{B_{0, k}\right\}$.

Since the neural network is used to approximate the unknown continuous function in this paper, $\phi_{0}\left(X_{1}\right)$ can be further rewritten as the following form

$$
\phi_{0}\left(X_{1}\right)=W_{0}^{* T} \psi_{0}\left(X_{1}\right)+B_{1}\left(X_{1}\right),
$$

where $W_{0}^{*}$ represents the unknown ideal weight vector. $\psi_{0}\left(X_{1}\right)$ represents the basic function vector and its dimension is $L_{0} . B_{1}\left(X_{1}\right)$ denotes the approximate error.

Using the inequality as shown in Proposition 1 and noting $\psi_{0}^{T}\left(X_{1}\right) \psi_{0}\left(X_{1}\right) \leq L_{0}$, we obtain $B_{0}^{L}\left|S_{1}\right| \phi_{0}\left(X_{1}\right) \leq \frac{B_{0}^{L} a_{12}^{\tau(1+\tau)}}{1+\tau}\left|S_{1}\right|^{1+\tau} \theta_{0}+\frac{B_{0}^{L} \tau}{(1+\tau) a_{12}^{\tau(1+\tau)}}+\frac{B_{0}^{L}}{1+\tau}\left|S_{1}\right|^{1+\tau}+\frac{B_{0}^{L} \tau}{1+\tau}\left|B_{1}\left(X_{1}\right)\right|^{\frac{1+\tau}{\tau}}$

Thus, there exists a continuous function $K_{1}\left(y, v, y_{r}, \dot{y}_{r}\right) \geq 0$ satisfying

$$
\frac{B_{0}^{L} \tau}{1+\tau}\left|B_{1}\left(X_{1}\right)\right|^{\frac{1+\tau}{\tau}} \leq K_{1}\left(y, v, y_{r}, \dot{y}_{r}\right)
$$

Substituting (38) into (39), and combining (40) with (41), we have

$$
\begin{aligned}
\dot{V}_{1} \leq & \frac{1}{2} y_{2}^{2}+B_{0}^{L} \frac{a_{11}^{\tau(1+\tau)}}{1+\tau}\left|S_{1}\right|^{1+\tau} \bar{\omega}^{*} \tilde{\theta}_{1}+B_{0}^{L} \frac{a_{12}^{\tau(1+\tau)}}{1+\tau}\left|S_{1}\right|^{1+\tau} \tilde{\theta}_{0}+\frac{2 B_{0}^{L} a_{13}^{\tau(1+\tau)} P_{m} S_{1}^{2(1+\tau)}}{\gamma_{\varepsilon} \varepsilon^{* 2}(1+\tau)} \tilde{H} \\
& +K_{0}\left(y, v, y_{r}, \bar{m}\right)-\frac{B_{0}^{L}}{\gamma_{\varepsilon}}\left(h-3-\gamma_{\varepsilon}\right) \varepsilon^{T} \varepsilon-\gamma_{0} B_{0}^{L} \tilde{\theta}_{0} \dot{\hat{\theta}}_{0}-\gamma_{1} B_{0}^{L} \tilde{\theta}_{1} \dot{\hat{\theta}}_{1}-\gamma_{H} B_{0}^{L} \tilde{H} \dot{\hat{H}}
\end{aligned}
$$




$$
\begin{aligned}
& +\frac{d}{\lambda^{*}}+\frac{5}{4 B_{0}^{L}}-c_{1} S_{1}^{2} B_{0}^{L}+\frac{\tau}{(1+\tau) a_{11}^{\tau(1+\tau)}\left(B_{0}^{L}\right)^{1 / \tau}}+\frac{2 \tau B_{0}^{L}}{\varepsilon^{* 2} \gamma_{\varepsilon}(1+\tau) a_{13}^{\tau(1+\tau)}}+\frac{1}{2} S_{2}^{2} \\
& +K_{1}\left(y, v, y_{r}, \dot{y}_{r}\right)-\frac{\bar{c}^{*}}{\lambda^{*}} v+\frac{\tau B_{0}^{L}}{(1+\tau) a_{12}^{\tau(1+\tau)}}
\end{aligned}
$$

where $K_{0}\left(y, v, y_{r}, \bar{m}\right)=B_{0}^{L}\left(1-\frac{S_{1}^{2}}{\varepsilon^{* 2}}\right)\left(Q(y, v)+\frac{2}{\gamma_{\varepsilon}}\left(P_{m} H\right)^{1 /(1+\tau)}\right)$.

The parameter adaptive laws are updated as follows

$$
\begin{gathered}
\dot{\hat{\theta}}_{0}=\frac{a_{12}^{\tau(1+\tau)}}{\gamma_{0}(1+\tau)} S_{1}^{1+\tau}-\frac{\bar{\lambda}_{0}}{\gamma_{0}} \hat{\theta}_{0}, \\
\dot{\hat{\theta}}_{1}=\frac{a_{11}^{\tau(1+\tau)}}{\gamma_{1}(1+\tau)} S_{1}^{1+\tau} \bar{\omega}^{*}-\frac{\bar{\lambda}_{1}}{\gamma_{1}} \hat{\theta}_{1}, \\
\dot{\hat{H}}=\frac{2 S_{1}^{2(1+\tau)} a_{13}^{\tau(1+\tau)}}{\gamma_{\varepsilon} \varepsilon^{* 2}(1+\tau)} P_{m}-\frac{\bar{\lambda}_{H}}{\gamma_{H}} \hat{H},
\end{gathered}
$$

where $\bar{\lambda}_{0}>0, \bar{\lambda}_{1}>0$ and $\bar{\lambda}_{H}>0$ are three design parameters.

Remark 5. Unlike those adopted in [18, 27-32], the adaptive laws (43)-(45) are not dependent upon the dynamical signal $v$, which means that the dynamical signal introduced is not required to be measured in this paper.

Substituting (43)-(45) into (42), we obtain

$$
\begin{aligned}
\dot{V}_{1} \leq & \frac{1}{2} y_{2}^{2}+\frac{1}{2} S_{2}^{2}-\frac{B_{0}^{L}}{\gamma_{\varepsilon}}\left(h-3-\gamma_{\varepsilon}\right) \varepsilon^{T} \varepsilon+B_{0}^{L} \bar{\lambda}_{0} \tilde{\theta}_{0} \hat{\theta}_{0}+B_{0}^{L} \bar{\lambda}_{1} \tilde{\theta}_{1} \hat{\theta}_{1}+B_{0}^{L} \bar{\lambda}_{H} \tilde{H} \hat{H}-c_{1} B_{0}^{L} S_{1}^{2} \\
& -\frac{\bar{c}^{*}}{\lambda^{*}} v+C_{1}+K_{1}\left(y, v, y_{r}, \dot{y}_{r}\right)+K_{0}\left(y, v, y_{r}, \bar{m}\right)
\end{aligned}
$$

where $C_{1}=\frac{d}{\lambda^{*}}+\frac{\tau}{(1+\tau) a_{11}^{\tau(1+\tau)}\left(B_{0}^{L}\right)^{1 / \tau}}+\frac{B_{0}^{L} \tau}{(1+\tau) a_{12}^{\tau(1+\tau)}}+\frac{2 B_{0}^{L} \tau}{\gamma_{\varepsilon} \varepsilon^{* 2}(1+\tau) a_{13}^{\tau(1+\tau)}}+\frac{5}{4 B_{0}^{L}}$.

Further, there exists a continuous function $B_{2}\left(\bar{S}_{2}, y_{2}, \varepsilon, \xi_{0}, \Xi, \bar{\lambda}_{m+2}, \hat{\theta}_{0}, \hat{\theta}_{1}, \hat{H}, \bar{m}, y_{r}, \dot{y}_{r}\right) \geq 0$ such that

$$
\left|\dot{y}_{2}+\frac{y_{2}}{\tau_{2}}\right| \leq B_{2}\left(\bar{S}_{2}, y_{2}, \varepsilon, \xi_{0}, \Xi, \bar{\lambda}_{m+2}, \hat{\theta}_{0}, \hat{\theta}_{1}, \hat{H}, \bar{m}, y_{r}, \dot{y}_{r}\right)
$$

$$
y_{2} \dot{y}_{2} \leq-\frac{y_{2}^{2}}{\tau_{2}}+\left|y_{2}\right| B_{2}\left(\bar{S}_{2}, y_{2}, \varepsilon, \xi_{0}, \Xi, \bar{\lambda}_{m+2}, \hat{\theta}_{0}, \hat{\theta}_{1}, \hat{H}, \bar{m}, y_{r}, \dot{y}_{r}\right) \leq-\frac{y_{2}^{2}}{\tau_{2}}+\frac{y_{2}^{2}}{2}+\frac{B_{2}^{2}}{2}
$$

where $\bar{S}_{2}=\left[S_{1}, S_{2}\right]^{T}$ and $\bar{\lambda}_{m+2}=\left[\lambda_{1}, \lambda_{2}, \cdots, \lambda_{m+2}\right]^{T}$. 
Step $i(2 \leq i \leq \rho-1)$. Define the $i$ th dynamic surface $S_{i}=\mu_{m, i}-w_{i}$, then

$$
\dot{S}_{i}=-l_{i} \mu_{m, 1}+u_{m, i+1}-\dot{w}_{i} .
$$

Choose a Lyapunov function candidate for inductive step as the following form

$$
V_{S_{i}}=\frac{1}{2} S_{i}^{2}
$$

Select the virtual control law as follows

$$
\alpha_{i}=-c_{i} S_{i}^{\tau}+l_{i} \mu_{m, 1}+\dot{w}_{i}-\frac{3}{2} S_{i}
$$

where $c_{i}>0$ is a design parameter.

Similar to the first step, a first-order filter is designed as follows

$$
\tau_{i+1} \dot{w}_{i+1}+w_{i+1}=\alpha_{i}, w_{i+1}(0)=\alpha_{i}(0)
$$

where $\tau_{i+1}>0$ is a constant. Let $y_{i+1}=w_{i+1}-\alpha_{i}$, then $\dot{w}_{i+1}=-\frac{y_{i+1}}{\tau_{i+1}}$.

Since $\mu_{m, i+1}=S_{i+1}+y_{i+1}+\alpha_{i}$, taking the derivate of $V_{S_{i}}$ and substituting (51) into it, we have

$$
\begin{aligned}
\dot{V}_{S_{i}} & \leq-S_{i} l_{i} \mu_{m, 1}+S_{i} \alpha_{i}-S_{i} \dot{\omega}_{i}+S_{i}^{2}+\frac{1}{2} S_{i+1}^{2}+\frac{1}{2} y_{i+1}^{2} \\
& \leq-c_{i} S_{i}^{1+\tau}+\frac{1}{2} S_{i+1}^{2}+\frac{1}{2} y_{i+1}^{2}-\frac{1}{2} S_{i}^{2} .
\end{aligned}
$$

Further, there exists a continuous function $B_{i+1}\left(\bar{S}_{i+1}, \bar{y}_{i+1}, \varepsilon, \xi_{0}, \Xi, \bar{\lambda}_{m+2}, \hat{\theta}_{0}, \hat{\theta}_{1}, \hat{H}, \bar{m}, y_{r}, \dot{y}_{r}\right) \geq$ 0 such that

$$
\begin{aligned}
\mid \dot{y}_{i+1}+ & \frac{y_{i+1}}{\tau_{i+1}} \mid \leq B_{i+1}\left(\bar{S}_{i+1}, \bar{y}_{i+1}, \varepsilon, \xi_{0}, \Xi, \bar{\lambda}_{m+2}, \hat{\theta}_{0}, \hat{\theta}_{1}, \hat{H}, \bar{m}, y_{r}, \dot{y}_{r}\right), \\
y_{i+1} \dot{y}_{i+1} & \leq-\frac{y_{i+1}^{2}}{\tau_{i+1}}+\left|y_{i+1}\right| B_{i+1}\left(\bar{S}_{i+1}, \bar{y}_{i+1}, \varepsilon, \xi_{0}, \Xi, \bar{\lambda}_{m+2}, \hat{\theta}_{0}, \hat{\theta}_{1}, \hat{H}, \bar{m}, y_{r}, \dot{y}_{r}\right) \\
& \leq-\frac{y_{i+1}^{2}}{\tau_{i+1}}+\frac{y_{i+1}^{2}}{2}+\frac{B_{i+1}^{2}}{2},
\end{aligned}
$$

where $\bar{y}_{j}=\left[y_{2}, y_{3}, \cdots, y_{j}\right]^{T}$ and $\bar{S}_{j}=\left[S_{1}, S_{2}, \cdots, S_{j}\right]^{T}, j=2,3, \cdots, \rho$.

Step $\rho$. Define the last dynamic surface $S_{\rho}=\mu_{m, \rho}-w_{\rho}$. The derivative of $S_{\rho}$ is represented as

$$
\dot{S}_{\rho}=-l_{\rho} \mu_{m, 1}+\mu_{m, \rho+1}+u-\dot{w}_{\rho} .
$$


Choose the following Lyapunov function candidate

$$
V_{S_{\rho}}=\frac{1}{2} S_{\rho}^{2}
$$

Select the control law as follows

$$
u=-c_{\rho} S_{\rho}^{\tau}+l_{\rho} \mu_{m, 1}-\mu_{m, \rho+1}+\dot{w}_{\rho}-\frac{1}{2} S_{\rho} .
$$

Taking the derivate of $V_{S_{\rho}}$ and substituting (57) into it, we have

$$
\dot{V}_{S_{\rho}}=-c_{\rho} S_{\rho}^{1+\tau}-\frac{1}{2} S_{\rho}^{2}
$$

\section{Stability analysis}

In this section, we will state our main results.

Define some compact sets as follows

$$
\begin{gathered}
\Omega_{1}=\left\{\left[S_{1}, \varepsilon^{T}, v, \hat{\theta}_{0}, \hat{\theta}_{1}, \hat{H}\right]^{T}: V_{1} \leq p\right\} \subset R^{p_{1}}, \\
\Omega_{i}=\left\{\left[\bar{S}_{i}^{T}, \bar{y}_{i}^{T}, \varepsilon^{T}, v, \hat{\theta}_{0}, \hat{\theta}_{1}, \hat{H}\right]^{T}: V_{i} \leq p\right\} \subset R^{p_{i}}, i=2,3, \cdots, \rho .
\end{gathered}
$$

where $p>0$ denotes an arbitrary given constant, $p_{i}=\rho+n+i+3, i=1,2, \cdots, \rho$, and

$$
V_{i}=V_{1}+\sum_{j=2}^{i} V_{S_{j}}+\frac{1}{2} \sum_{j=2}^{i} y_{j}^{2}
$$

Theorem 1. Consider the closed-loop system consisting of (1),(2) under Assumptions 1-5, control law (57), and adaptive laws (43)-(45). For any bounded initial conditions, there exist constants $h, c_{l}, \tau_{i}, \gamma_{\varepsilon}, \gamma_{0}, \gamma_{1}, \gamma_{H}$ satisfying $V\left(t_{0}\right) \leq p$ and

$$
\left\{\begin{array}{l}
h \geq 3+\gamma_{\varepsilon}+\tilde{c} \lambda_{\max }(P), \\
\frac{1}{\tau_{i}} \geq \frac{\tilde{c}}{2}+1, i=2,3, \cdots, \rho,
\end{array}\right.
$$

such that the tracking error converges to a small neighbourhood of zero in finite time under arbitrary switchings, where $\tilde{c}>0$ is a parameter satisfying $\tilde{c} \leq \min _{l=1,2, \cdots, \rho}\left\{c_{l}\right\}$.

Proof. Consider the following Lyapunov function candidate

$$
V=V_{1}+\sum_{i=2}^{\rho} V_{S_{i}}+\frac{1}{2} \sum_{i=2}^{\rho} y_{i}^{2} .
$$


If $V \leq p$, then $V_{i} \leq p$. From aforementioned compact sets, we obtain that $\bar{S}_{\rho}, y, \bar{y}_{\rho}, \varepsilon, v, \hat{\theta}_{0}, \hat{\theta}_{1}$ and $\hat{H}$ are all bounded. Further, the boundedness of the other signals will be proved on this basis. According to (44) and (45), we have that $\bar{\omega}^{*}, \bar{m}$ are bounded. Noting (22) and (25), we obtain that $\xi_{0}, \Xi, \mu_{m-1,2}, \cdots, \mu_{1,2}, \mu_{0,2}$ are also bounded. In view of (19), we have $\dot{\mu}_{0,1}=-l_{1} \mu_{0,1}+\mu_{0,2}$, thus, $\mu_{0,1}$ is bounded. Furthermore, we have

$$
\left[\begin{array}{c}
\mu_{0,1} \\
\mu_{0,2} \\
\mu_{1,2} \\
\mu_{2,2} \\
\vdots \\
\mu_{m-1,2}
\end{array}\right]=\left[\begin{array}{cccccc}
1 & 0 & 0 & 0 & \cdots & 0 \\
0 & 1 & 0 & 0 & \cdots & 0 \\
-l_{2} & 0 & 1 & 0 & \cdots & 0 \\
* & -l_{2} & 0 & 1 & \cdots & 0 \\
\vdots & \vdots & \vdots & \vdots & \ddots & \vdots \\
* & * & * & * & \cdots & 1
\end{array}\right]\left[\begin{array}{c}
\lambda_{1} \\
\lambda_{2} \\
\lambda_{3} \\
\lambda_{4} \\
\vdots \\
\lambda_{m+1}
\end{array}\right] .
$$

According to (61), $\lambda_{1}, \lambda_{2}, \cdots, \lambda_{m+1}$ are all bounded. From (21), we obtain that $\mu_{m, 1}$ is bounded. Noting (19), we have $\dot{\mu}_{m, 1}=-l_{1} \mu_{m, 1}+\mu_{m, 2}$, which implies $\mu_{m, 2}$ is bounded. Since $\mu_{m, 2}=S_{2}+\alpha_{1}+y_{2}$, it yields $\alpha_{1}$ is bounded. In view of the first-order filters, we obtain that $\dot{w}_{2}, \dot{w}_{3}, \cdots, \dot{w}_{\rho}$ are all bounded. Further, $\alpha_{i}, i=2,3, \cdots, \rho-1$, is also bounded. Because $\mu_{m, i+1}=S_{i+1}+y_{i+1}+\alpha_{i}, i=1,2, \cdots, \rho-1, \mu_{m, 3}, \mu_{m, 4}, \cdots, \mu_{m, \rho}$ are all bounded. From (21), we obtain that $\lambda_{m+2}, \lambda_{m+3}, \cdots, \lambda_{m}$ are also bounded, that is, $\lambda$ is bounded. In view of K-filters (22), we obtain that $\mu_{0}, \mu_{1}, \cdots, \mu_{m}$ are bounded. Noting (4), (18), (23) and (57), we have that $u, \Omega, \hat{x}, z, x$ are all bounded. Therefore, all closed-loop signals are bounded.

Furthermore, it is easy to know that $\Omega_{1} \times R^{p_{\rho}-p_{1}} \supset \Omega_{2} \times R^{p_{\rho}-p_{2}} \supset \cdots \supset \Omega_{\rho-1} \times R^{p_{\rho}-p_{\rho-1}} \supset$ $\Omega_{\rho}$. Due to the boundedness of $\lambda, \bar{m}$, continuous functions $K_{1}(\cdot)$ and $\left|K_{0}(\cdot)\right|$ have maximums $K_{1}^{M}$ and $N_{1}$ on the compact set $\Omega_{r} \times \Omega_{1}$, respectively. $B_{i}(\cdot), i=2,3, \cdots, \rho$, has a maximum $B_{i}^{M}$ on the compact set $\Omega_{r} \times \Omega_{i}$.

Taking the derivative of (60) and using (46), (52), (58), we obtain

$$
\begin{aligned}
\dot{V} \leq & -\tilde{c} B_{0}^{L} S_{1}^{2}-\sum_{i=2}^{\rho} \tilde{c} S_{i}^{1+\tau}-\frac{B_{0}^{L}}{\gamma_{\varepsilon}} \tilde{c} \varepsilon^{T} P \varepsilon+B_{0}^{L} \bar{\lambda}_{0} \tilde{\theta}_{0} \hat{\theta}_{0}+B_{0}^{L} \bar{\lambda}_{1} \tilde{\theta}_{1} \hat{\theta}_{1}+B_{0}^{L} \bar{\lambda} H \tilde{H} \hat{H}+K_{1}^{M} \\
& -\frac{\tilde{c}}{2} \sum_{i=2}^{\rho} y_{i}^{2}+C_{1}+N_{1}-\frac{\bar{c}^{*}}{\lambda^{*}} v-2^{(1+\tau) / 2} \tilde{c}\left(\frac{1}{2} S_{1}^{2}\right)^{(1+\tau) / 2}+2^{(1+\tau) / 2} \tilde{c}\left(\frac{1}{2} S_{1}^{2}\right)^{(1+\tau) / 2} \\
& +2^{(1+\tau) / 2} \tilde{c}\left(\frac{v}{\lambda^{*}}\right)^{(1+\tau) / 2}-2^{(1+\tau) / 2} \tilde{c}\left(\frac{v}{\lambda^{*}}\right)^{(1+\tau) / 2}+\frac{1}{2} \sum_{i=2}^{\rho}\left(B_{i}^{M}\right)^{2}-2^{(1+\tau) / 2} \tilde{c}\left(\varepsilon^{T} P \varepsilon\right) \\
& +2^{(1+\tau) / 2} \tilde{c}\left(\varepsilon^{T} P \varepsilon\right)-2^{(1+\tau) / 2} \tilde{c} \sum_{i=2}^{\rho}\left(\frac{1}{2} y_{i}^{2}\right)^{(1+\tau) / 2}+2^{(1+\tau) / 2} \tilde{c} \sum_{i=2}^{\rho}\left(\frac{1}{2} y_{i}^{2}\right)^{(1+\tau) / 2} .
\end{aligned}
$$

Using the inequality as shown in Proposition 1, we have 


$$
\begin{gathered}
2^{(1+\tau) / 2} \tilde{c}\left(\frac{1}{2} S_{1}^{2}\right)^{(1+\tau) / 2}=\left(\tilde{c} B_{0}^{L} S_{1}^{2}\right)^{(1+\tau) / 2}\left(\frac{\tilde{c}^{(1-\tau) /(1+\tau)}}{B_{0}^{L}}\right)^{\frac{(1+\tau)(1-\tau)}{2(1-\tau)}} \leq \tilde{c} B_{0}^{L} S_{1}^{2}+\frac{1-\tau}{2} \tilde{c}\left(\frac{1}{B_{0}^{L}}\right)^{\frac{1+\tau}{1-\tau}} \\
2^{(1+\tau) / 2} \tilde{c}\left(\frac{v}{\lambda^{*}}\right)^{(1+\tau) / 2}=\left(\frac{\bar{c}^{*}}{\lambda^{*}} v\right)^{(1+\tau) / 2}\left(\frac{2 \tilde{c}^{2 /(1+\tau)}}{\bar{c}^{*}}\right)^{\frac{(1+\tau)(1-\tau)}{2(1-\tau)}} \leq \frac{\bar{c}^{*}}{\lambda^{*}} v+\frac{1-\tau}{2}\left(\frac{2 \tilde{c}^{2 /(1+\tau)}}{\bar{c}^{*}}\right)^{\frac{1+\tau}{1-\tau}} \\
2^{(1+\tau) / 2} \tilde{c} \sum_{i=2}^{\rho}\left(\frac{1}{2} y_{i}^{2}\right)^{(1+\tau) / 2}=\sum_{i=2}^{\rho}\left(\frac{1}{2} \tilde{c} y_{i}^{2}\right)^{(1+\tau) / 2}\left(2 \tilde{c}^{\frac{1-\tau}{1+\tau}}\right)^{\frac{(1+\tau)(1-\tau)}{2(1-\tau)}} \leq \frac{1}{2} \sum_{i=2}^{\rho} \tilde{c} y_{i}^{2}+\frac{1-\tau}{2} 2^{\frac{1+\tau}{1-\tau}} \sum_{i=2}^{\rho} \tilde{c} \\
2^{(1+\tau) / 2} \tilde{c}\left(\varepsilon^{T} P \varepsilon\right)^{(1+\tau) / 2}=\left(\frac{B_{0}^{L}}{\gamma_{\varepsilon}} \tilde{c} \varepsilon^{T} P \varepsilon\right)^{(1+\tau) / 2}\left(\frac{2 \tilde{c}^{(1+\tau) /(1-\tau)}}{B_{0}^{L}}\right)^{\frac{(1+\tau)(1-\tau)}{2(1-\tau)}} \\
\leq \frac{B_{0}^{L}}{\gamma_{\varepsilon}} \tilde{c} \varepsilon^{T} P \varepsilon+\frac{1-\tau}{2} \tilde{c}\left(\frac{2}{B_{0}^{L}}\right)^{\frac{1+\tau}{1-\tau}} .
\end{gathered}
$$

Further, substituting (63) into (62), we obtain

$$
\begin{aligned}
\dot{V} \leq & -2^{(1+\tau) / 2} \tilde{c} \sum_{i=1}^{\rho}\left(\frac{1}{2} S_{i}^{2}\right)^{(1+\tau) / 2}+B_{0}^{L} \bar{\lambda}_{0} \tilde{\theta}_{0} \hat{\theta}_{0}+B_{0}^{L} \bar{\lambda}_{1} \tilde{\theta}_{1} \hat{\theta}_{1}+B_{0}^{L} \bar{\lambda}_{H} \tilde{H} \hat{H}+C \\
& -2^{(1+\tau) / 2} \tilde{c} \sum_{i=2}^{\rho}\left(\frac{1}{2} y_{i}^{2}\right)^{(1+\tau) / 2}-2^{(1+\tau) / 2} \tilde{c}\left(\varepsilon^{T} P \varepsilon\right)^{(1+\tau) / 2}-2^{(1+\tau) / 2} \tilde{c}\left(\frac{v}{\lambda^{*}}\right)^{(1+\tau) / 2},
\end{aligned}
$$

where $C=C_{1}+N_{1}+\frac{1}{2} \sum_{i=2}^{\rho}\left(B_{i}^{M}\right)^{2}+\frac{1-\tau}{2} \tilde{c}\left(\frac{1}{B_{0}^{L}}\right)^{\frac{1+\tau}{1-\tau}}+\frac{1-\tau}{2}\left(\frac{2 \tilde{c}^{2 /(1+\tau)}}{\bar{c}^{*}}\right)^{\frac{1+\tau}{1-\tau}}+\frac{1-\tau}{2} \tilde{c} \sum_{i=2}^{\rho} 2^{\frac{1+\tau}{1-\tau}}+$ $\frac{1-\tau}{2} \tilde{c}\left(\frac{2}{B_{0}^{L}}\right)^{\frac{1+\tau}{1-\tau}}+K_{1}^{M}$

Employing Proposition 1, one gets

$$
B_{0}^{L} \bar{\lambda}_{0} \tilde{\theta}_{0} \hat{\theta}_{0} \leq B_{0}^{L} \bar{\lambda}_{0}\left(-\tilde{\theta}_{0}^{2}+\frac{1}{2 \bar{c}} \tilde{\theta}_{0}^{2}+\frac{\bar{c}}{2} \theta_{0}^{2}\right) \leq \frac{-\bar{\lambda}_{0} B_{0}^{L}(2 \bar{c}-1)}{2 \bar{c}} \tilde{\theta}_{0}^{2}+\frac{\bar{\lambda}_{0} \bar{c}}{2} B_{0}^{L} \theta_{0}^{2},
$$

where $\bar{c}$ is a design parameter satisfying $\bar{c}>\frac{1}{2}$.

Furthermore, we have

$$
\begin{gathered}
B_{0}^{L} \bar{\lambda}_{1} \tilde{\theta}_{1} \hat{\theta}_{1} \leq \frac{-\bar{\lambda}_{1} B_{0}^{L}(2 \bar{c}-1)}{2 \bar{c}} \tilde{\theta}_{1}^{2}+\frac{\bar{\lambda}_{1} \bar{c}}{2} B_{0}^{L} \theta_{1}^{2}, \\
B_{0}^{L} \bar{\lambda}_{H} \tilde{H} \hat{H} \leq \frac{-\bar{\lambda}_{H} B_{0}^{L}(2 \bar{c}-1)}{2 \bar{c}} \tilde{H}^{2}+\frac{\bar{\lambda}_{H} \bar{c}}{2} B_{0}^{L} H^{2} .
\end{gathered}
$$

Applying Proposition 1 again, we obtain

$$
\begin{aligned}
& 2^{(1+\tau) / 2} \tilde{c}\left(\frac{\gamma_{0}}{2} B_{0}^{L} \tilde{\theta}_{0}^{2}\right)^{(1+\tau) / 2}=\left(\frac{\bar{\lambda}_{0}(2 \bar{c}-1) \tilde{\theta}_{0}^{2} B_{0}^{L}}{2 \bar{c}}\right)^{(1+\tau) / 2}\left(\frac{2 \bar{c} \gamma_{0} \tilde{c}^{2 /(1+\tau)}}{\bar{\lambda}_{0}(2 \bar{c}-1)}\right)^{\frac{(1+\tau)(1-\tau)}{2(1-\tau)}} \\
& \leq \frac{(2 \bar{c}-1) \bar{\lambda}_{0} B_{0}^{L} \tilde{\theta}_{0}^{2}}{2 \bar{c}}+\frac{1-\tau}{2}\left(\frac{2 \bar{c} \gamma_{0} \tilde{c}^{2 /(1+\tau)}}{\bar{\lambda}_{0}(2 \bar{c}-1)}\right)^{\frac{1+\tau}{1-\tau}} \\
& 18
\end{aligned}
$$


Similar to (67), we have

$$
\begin{aligned}
& 2^{(1+\tau) / 2} \tilde{c}\left(\frac{\gamma_{1}}{2} B_{0}^{L} \tilde{\theta}_{1}^{2}\right)^{(1+\tau) / 2} \leq \frac{(2 \bar{c}-1) \bar{\lambda}_{1} B_{0}^{L} \tilde{\theta}_{1}^{2}}{2 \bar{c}}+\frac{1-\tau}{2}\left(\frac{2 \bar{c} \gamma_{0} \tilde{c}^{2 /(1+\tau)}}{\bar{\lambda}_{1}(2 \bar{c}-1)}\right)^{\frac{1+\tau}{1-\tau}}, \\
& 2^{(1+\tau) / 2} \tilde{c}\left(\frac{\bar{\gamma}_{H}}{2} B_{0}^{L} \tilde{H}^{2}\right)^{(1+\tau) / 2} \leq \frac{(2 \bar{c}-1) \bar{\lambda}_{H} B_{0}^{L} \tilde{H}^{2}}{2 \bar{c}}+\frac{1-\tau}{2}\left(\frac{2 \bar{c} \gamma_{H} \tilde{c}^{2 /(1+\tau)}}{\bar{\lambda}_{H}(2 \bar{c}-1)}\right)^{\frac{1+\tau}{1-\tau}} .
\end{aligned}
$$

Further, substituting (65)-(68) into (64), we get

$$
\begin{aligned}
\dot{V} \leq & -2^{(1+\tau) / 2} \tilde{c} \sum_{i=1}^{\rho}\left(\frac{1}{2} S_{i}^{2}\right)^{(1+\tau) / 2}-2^{(1+\tau) / 2} \tilde{c}\left(\frac{B_{0}^{L}}{\gamma_{\varepsilon}} \varepsilon^{T} P \varepsilon\right)^{(1+\tau) / 2}-2^{(1+\tau) / 2} \tilde{c}\left(\frac{\gamma_{0}}{2} B_{0}^{L} \tilde{\theta}_{0}^{2}\right)^{(1+\tau) / 2} \\
& -2^{(1+\tau) / 2} \tilde{c}\left(\frac{\gamma_{0}}{2} B_{0}^{L} \tilde{\theta}_{1}^{2}\right)^{(1+\tau) / 2}-2^{(1+\tau) / 2} \tilde{c}\left(\frac{\gamma_{H}}{2} B_{0}^{L} \tilde{H}^{2}\right)^{(1+\tau) / 2}-2^{(1+\tau) / 2} \tilde{c}\left(\frac{v}{\lambda^{*}}\right)^{(1+\tau) / 2} \\
& -2^{(1+\tau) / 2} \tilde{c} \sum_{i=2}^{\rho}\left(\frac{1}{2} y_{i}^{2}\right)^{(1+\tau) / 2}+d^{*}
\end{aligned}
$$

where $d^{*}=C+\frac{1-\tau}{2}\left(\frac{2 \bar{c} \gamma_{0} \tilde{c}^{2 /(1+\tau)}}{\bar{\lambda}_{0}(2 \bar{c}-1)}\right)^{\frac{1+\tau}{1-\tau}}+\frac{1-\tau}{2}\left(\frac{2 \bar{c} \gamma_{0} \tilde{c}^{2 /(1+\tau)}}{\bar{\lambda}_{1}(2 \bar{c}-1)}\right)^{\frac{1+\tau}{1-\tau}}+\frac{1-\tau}{2}\left(\frac{2 \bar{c} \gamma_{H} \tilde{c}^{2 /(1+\tau)}}{\bar{\lambda}_{H}(2 \bar{c}-1)}\right)^{\frac{1+\tau}{1-\tau}}+$ $\frac{\bar{\lambda}_{0} \bar{c}}{2} B_{0}^{L} \theta_{0}^{2}+\frac{\bar{\lambda}_{1} \bar{c}}{2} B_{0}^{L} \theta_{1}^{2}+\frac{\bar{\lambda}_{H} \bar{c}}{2} B_{0}^{L} H^{2}$.

Combining (69) with Proposition 3, we have

$$
\dot{V} \leq-c_{0} V^{(1+\tau) / 2}+d^{*}
$$

where $c_{0}=2^{(1+\tau) / 2} \tilde{c}$.

Since $0<\frac{1+\tau}{2}<1$, according to Lemma $3, \hat{\theta}_{0}, \hat{\theta}_{1}, \hat{H}, \bar{S}_{\rho}, v, \varepsilon$ and $\bar{y}_{\rho}$ can converge to a small neighbourhood of zero in finite time under arbitrary switchings, and we have

$$
V^{(1+\tau) / 2} \leq \frac{d^{*}}{c_{0}(1-\eta)}, \forall t \geq T^{*}=\frac{2 V^{(1-\tau) / 2}\left(t_{0}\right)}{c_{0} \eta(1-\tau)}
$$

where $0<\eta<1$.

When $V(t)=p$, we have $p_{0}=p^{(1+\tau) / 2}=V^{(1+\tau) / 2}(t)$. Select $c_{0}$ satisfying $c_{0}>\frac{d^{*}}{(1-\eta) p_{0}}$, then, we obtain from (70) that $\dot{V} \leq 0, \forall t \in\left[t_{0}, T^{*}\right)$. Since $V\left(t_{0}\right) \leq p$, we get $V(t) \leq p, \forall t \in$ $\left[t_{0}, T^{*}\right)$. Since $c_{0}>\frac{d^{*}}{(1-\eta) p_{0}}$, it follows from $(71)$ that $V^{(1+\tau) / 2}<p_{0}$, i.e., $V(t)<p, \forall t \geq T^{*}$.

The proof of Theorem 1 is completed. 
Remark 6. It can be seen from (59) that $\tilde{c}$ is independent of $\bar{c}^{*}$. Since $c_{l}, l=1,2, \cdots, \rho$, is freely selected by the designers, $\tilde{c}$ can be taken arbitrary large, which makes the tracking error and parameter estimation errors arbitrary small.

Remark 7. According to (59) and (69), we know that positive constants $c_{0}, d^{*}$ are determined by these parameters $\tau, \gamma_{\varepsilon}, \gamma_{0}, \gamma_{1}, \gamma_{H}, \bar{\lambda}_{0}, \bar{\lambda}_{1}, \bar{\lambda}_{H}, \varepsilon^{*}, c_{l}, l=1,2, \cdots, \rho$, and $\tau_{i}, i=$ $2,3, \cdots, \rho$. In what follows, we will give some suggestions in choosing these parameters:

(i) Choosing $\tau$ close to 1 helps to reduce $d^{*}$.

(ii) Increasing $\tau_{i}, c_{l}$ helps to increase $c_{0}$.

(iii) Decreasing $\bar{\lambda}_{0}, \bar{\lambda}_{1}, \bar{\lambda}_{H}, \gamma_{0}, \gamma_{1}$ and $\gamma_{H}$ helps to reduce $d^{*}$.

(iv) Increasing $\gamma_{\varepsilon}, \varepsilon^{*}$ helps to reduce $d^{*}$.

In practical cases, choosing appropriate design parameters will make the system show better performance. But the valid parameters can only be obtained after several attempts.

\section{Simulation Example}

In this section, the effectiveness of the control scheme proposed in this paper will be expressed. Consider the switched nonlinear systems with state and input unmodeled dynamics consisting of two subsystems:

\section{Subsystem 1:}

$$
\begin{aligned}
& \left\{\begin{array}{l}
\dot{z}=-4 z+x_{1}^{2} \sin \left(x_{1}\right) \\
\dot{x}_{1}=x_{2}+\frac{x_{1}-x_{1}^{3}}{1+x_{1}^{2}}+z^{2} \sin \left(x_{1}\right)+\sin ^{2}\left(x_{1}\right), \\
\dot{x}_{2}=x_{1}^{2} \tanh \left(x_{1}\right)-\left(x_{1}^{2}+2 x_{1}\right) \sin \left(x_{1}\right)+z^{2} \arctan \left(x_{1}\right)+x_{1}^{3} \sin ^{2}\left(x_{1}\right)+v \\
y=x_{1} .
\end{array}\right. \\
& \left\{\begin{array}{l}
\dot{\xi}=\arctan (\xi) \cos ^{2}(\xi)+u \\
v=3 \xi \operatorname{arccot}^{2}(\xi)+2 u .
\end{array}\right.
\end{aligned}
$$

\section{Subsystem 2:}

$$
\begin{aligned}
& \left\{\begin{array}{l}
\dot{z}=-4 z+x_{1}^{2} \sin \left(x_{1}\right) \\
\dot{x}_{1}=x_{2}+\frac{2\left(x_{1}+x_{1}^{2}\right)}{1+x_{1}^{4}} \sin \left(x_{1}\right)+\tanh \left(x_{1}\right) x_{1}^{3}+z^{2}+x_{1}^{2} \sin ^{2}(z), \\
\dot{x}_{2}=x_{1} \sin \left(x_{1}\right)+\sinh \left(x_{1}\right) \cosh \left(x_{1}\right)+z^{2} \sin (z)+\cos ^{2}\left(x_{1}\right) \sin ^{2}\left(x_{1}\right)+v \\
y=x_{1} .
\end{array}\right. \\
& \qquad \begin{array}{l}
\dot{\xi}=\xi \operatorname{arccot}(\xi) \sin ^{2}(\xi)+3 u \\
v=2 \xi \arctan ^{3}(\xi)+3 u .
\end{array}
\end{aligned}
$$


Furthermore, the desired tracking trajectory is taken as $y_{r}=0.25 \sin (0.5 t) \cos (0.5 t)$, and the dynamic signal is taken as the following form

$$
\dot{v}=-3 v+0.2 \sin \left(|y|^{2}\right)+10^{-3} .
$$

From (22), the filters are designed as follows

$$
\left\{\begin{array}{l}
\dot{\xi}_{1}=-l_{1} \xi_{1}+l_{2} \xi_{2}+l_{1} y, \\
\dot{\xi}_{2}=-l_{1} \xi_{1}+l_{2} y, \\
\dot{\Xi}_{(1)}=\left[-l_{1}, 1\right] \Xi+\left[G_{1}^{T}(y) 0_{1 \times 15}\right], \\
\dot{\Xi}_{(2)}=\left[-l_{2}, 1\right] \Xi+\left[0_{1 \times 15} G_{2}^{T}(y)\right], \\
\dot{\lambda}_{1}=-l_{1} \lambda_{1}+\lambda_{2}, \\
\dot{\lambda}_{2}=-l_{2} \lambda_{1}+u .
\end{array}\right.
$$

The finite-time controller for simulation object is designed as the following form

$$
u=-c_{2} S_{2}^{\tau}+l_{2} \mu_{0,1}+\dot{w}_{2}-\frac{1}{2} S_{2} .
$$

In this example, the design parameters are chosen as $\tau=3 / 7, c_{1}=0.1, c_{2}=0.2, l_{1}=$ $4, l_{2}=4, \tau_{2}=15, a_{11}=a_{13}=0.35, a_{12}=0.25, \gamma_{0}=0.3, \gamma_{1}=0.4, \gamma_{H}=\gamma_{\varepsilon}=0.2, \bar{\lambda}_{0}=$ $0.3, \bar{\lambda}_{1}=\bar{\lambda}_{H}=0.2, \varepsilon^{*}=5$, the initial conditions are selected as $x_{1}(0)=0.2, x_{2}(0)=$ $-0.2, \xi_{0}(0)=[1,1]^{T}, \lambda(0)=[0,0]^{T}, \Xi(0)=[1, \cdots 1]^{T} \in R^{60}, \hat{\theta}_{0}(0)=\hat{H}(0)=0.2, \hat{\theta}_{1}(0)=$ $0.3, z(0)=0.2, \xi(0)=0.2, v(0)=0.4, \bar{m}(0)=w_{2}(0)=0.2$. Then, combining K-filters $(22)$, adaptive laws (43)-(45), and control input (57), the simulation results are shown in Figs.1-8.

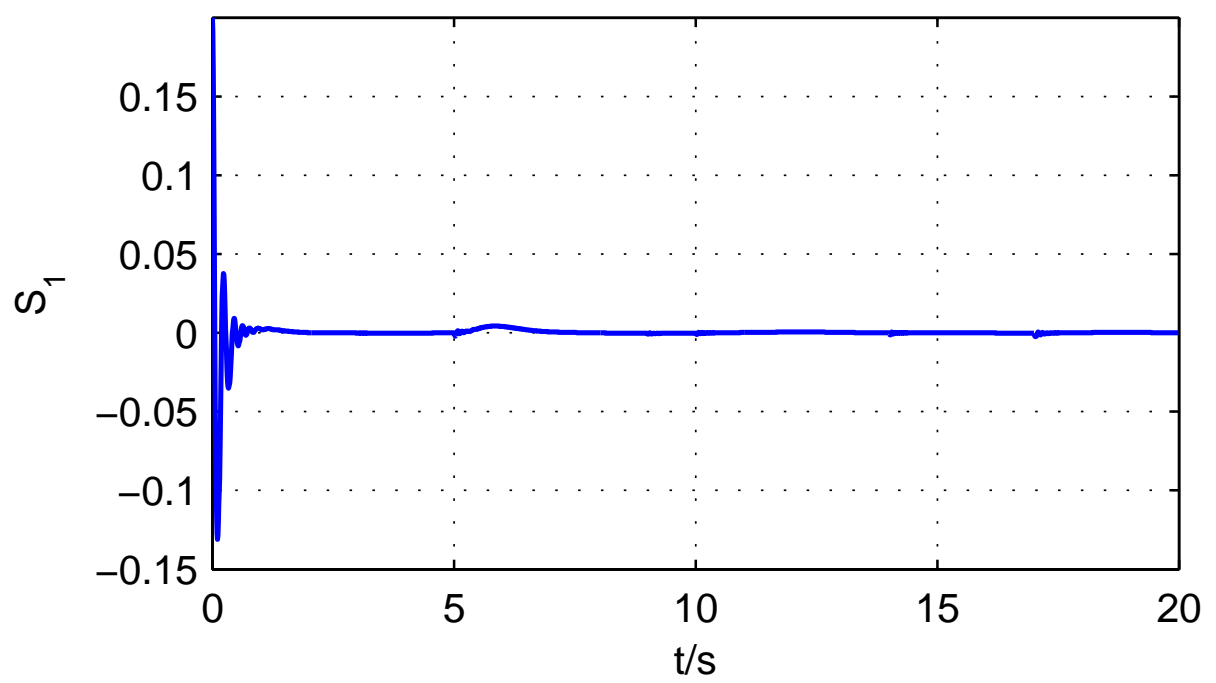

Figure 1: Tracking error $S_{1}$

Simulation results show that the tracking error converges to a small neighbourhood of zero in finite time under arbitrary switchings. Thus, the validity of the proposed control strategy is demonstrated. 


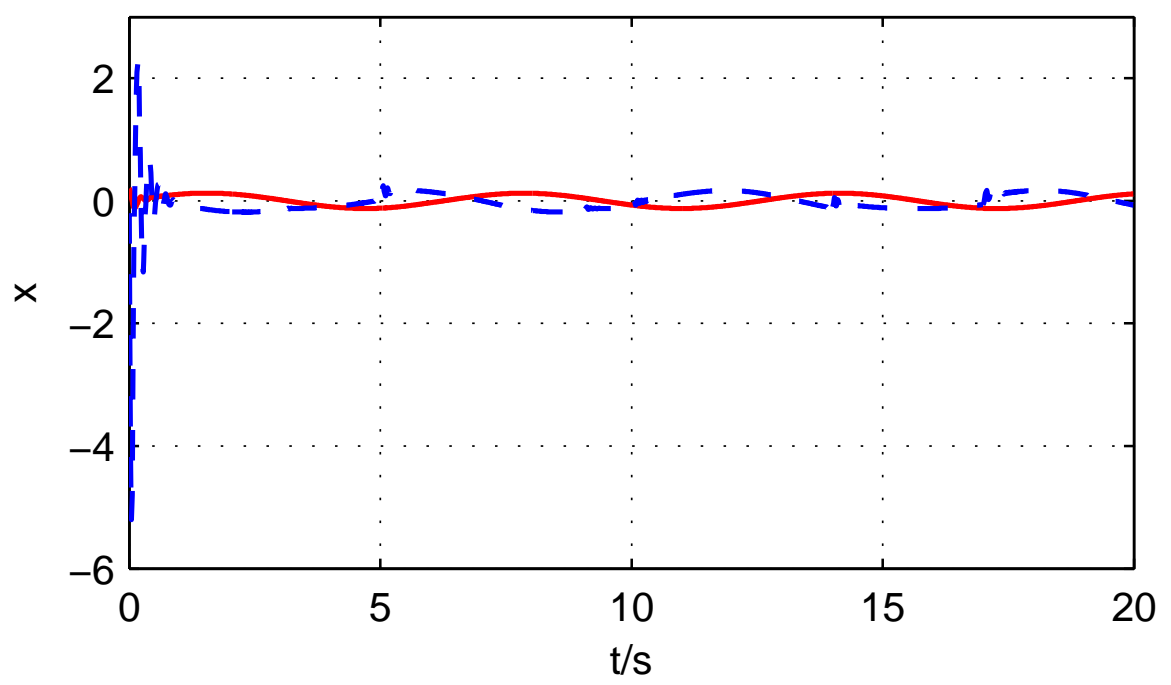

Figure 2: Trajectories of $x_{1}$ (solid line) and $x_{2}$ (dotted line)

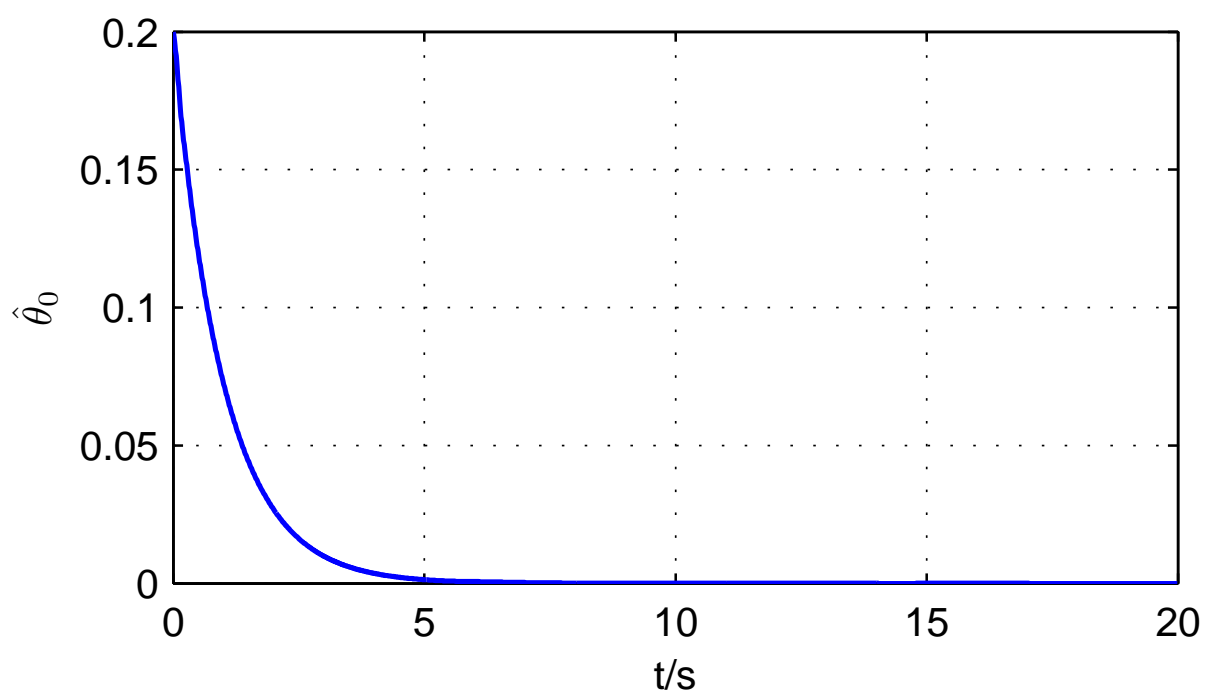

Figure 3: Estimated parameter $\hat{\theta}_{0}$

\section{Conclusions}

Combining DSC with K-filters, finite-time tracking control problem for a class of switched nonlinear systems with state and input unmodeled dynamics has been solved in this paper. By introducing a dynamical signal and a specific filter, unmodeled dynamics have been effectively dealt with. During the controller design, neural networks are used to approximate unknown continuous functions. Finally, it is proved that the tracking error converges to a small neighbourhood of zero in finite time under arbitrary switchings, and a numerical simulation is presented to show the feasibility and validity of the proposed method. In our 


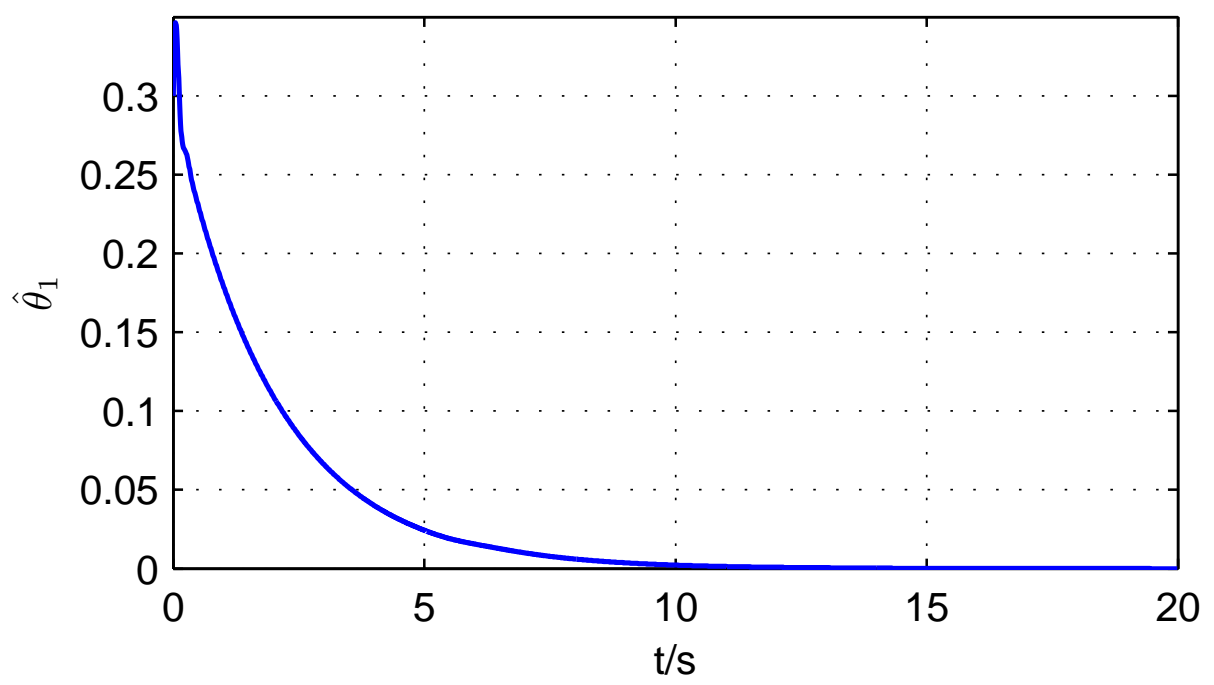

Figure 4: Estimated parameter $\hat{\theta}_{1}$

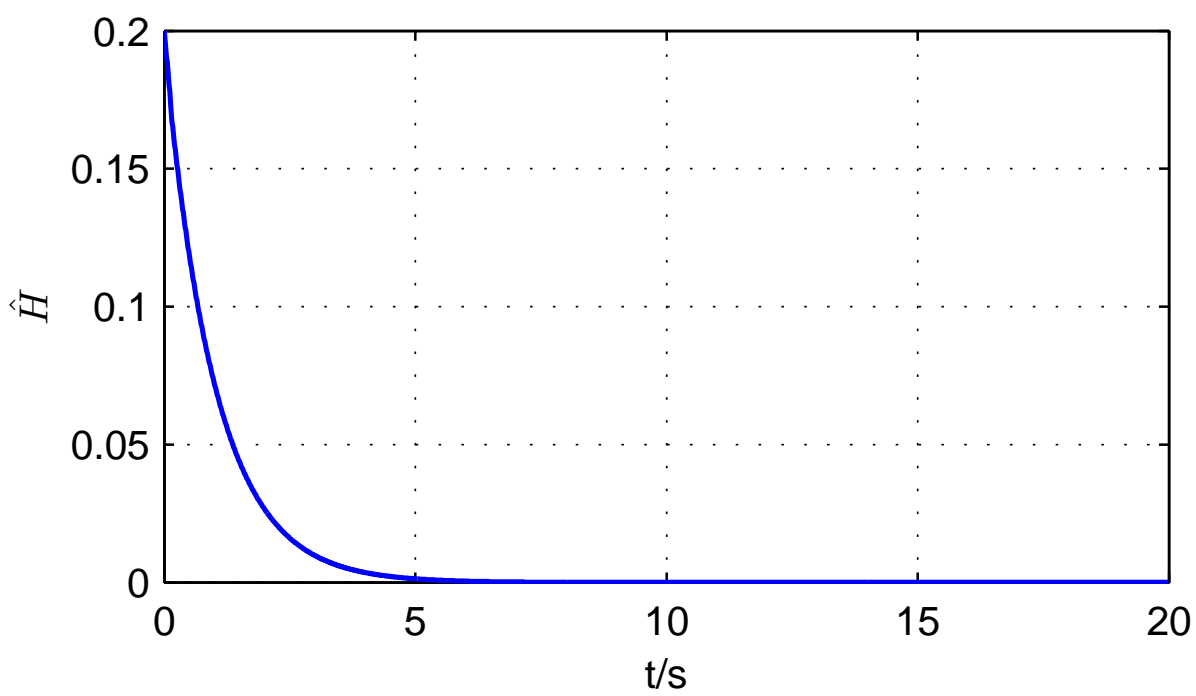

Figure 5: Estimated parameter $\hat{H}$

further work, with the aid of the existing results [45-47], fault-tolerant control for switched nonlinear systems with unmodeled dynamics may be considered.

\section{Acknowledgement}

This work was supported by the National Science Foundation of China under Grant no. 61273120 . 


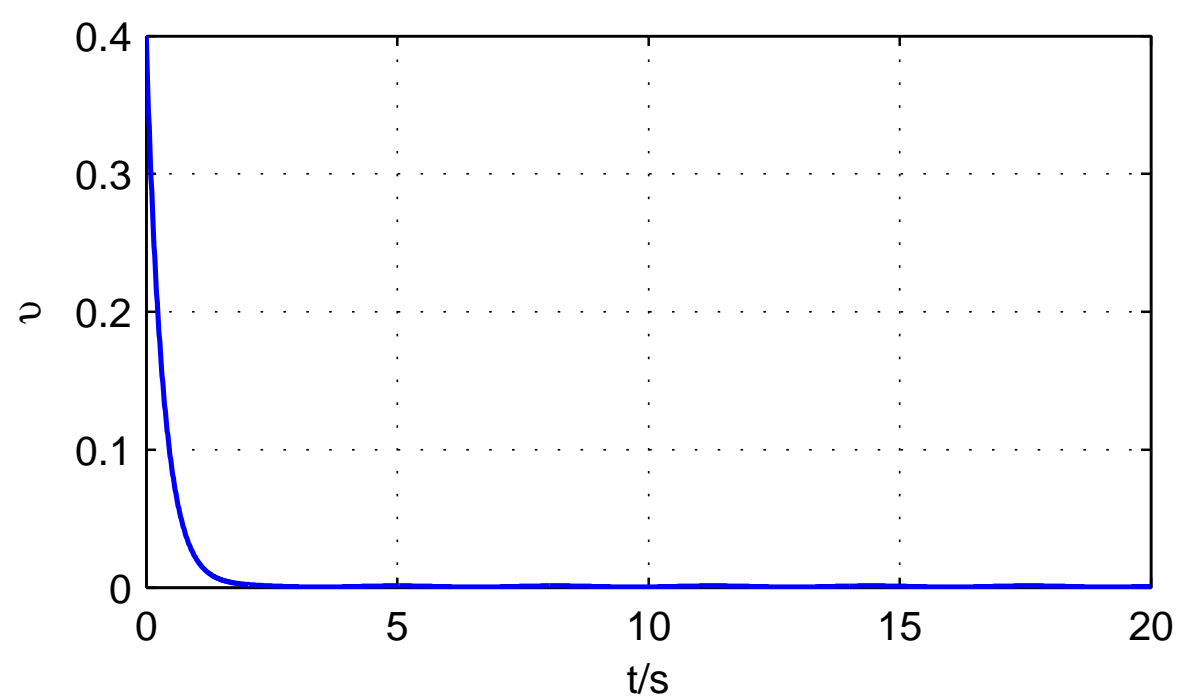

Figure 6: Dynamic signal $v$

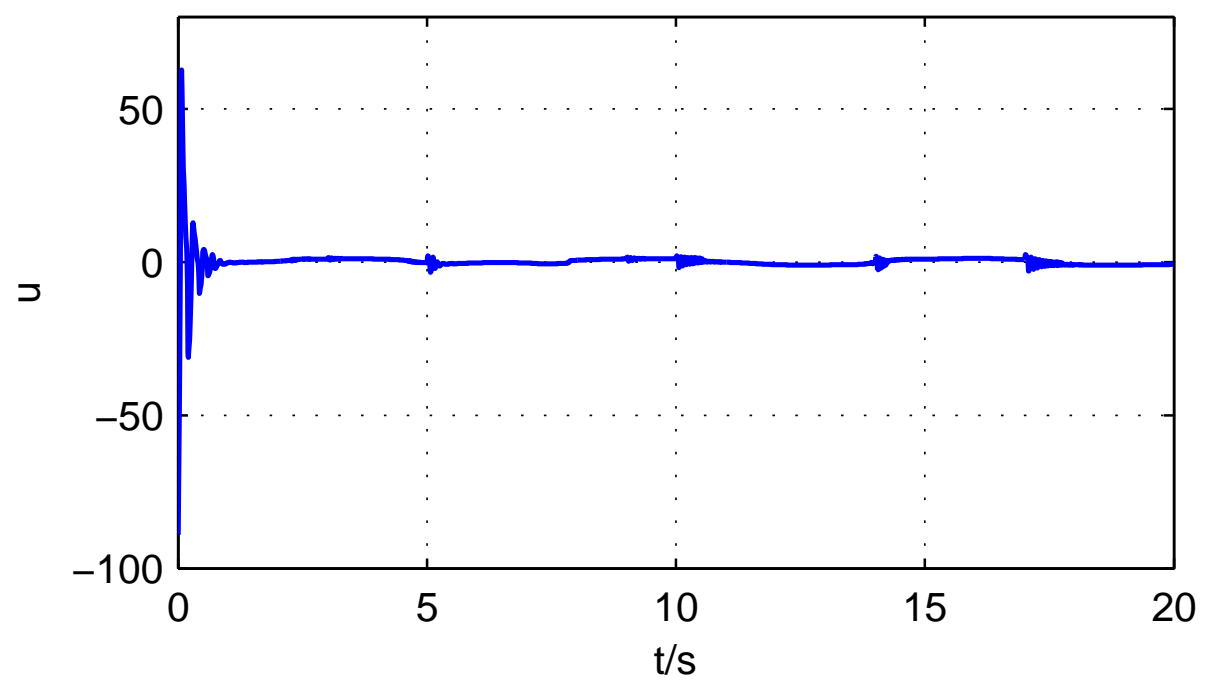

Figure 7: Control law $u$

\section{References}

[1] K. Lu, Y. Xia, Finite-time attitude stabilization for rigid spacecraft, International Journal of Robust and Nonlinear Control 25 (1) (2015) 32-51.

[2] L. Y. Sun, J. Zhao, Nonlinear adaptive control for the turbine steam valve with input constraints, Control Theory and Applications 26 (6) (2009) 601-606.

[3] B. Ding, J. Zhou, Y. Zhang, Multiple models control for attitude changing of a post-boost vehicle, Aerospace Control 26 (2) (2008) 47-51.

[4] Y. Yang, D. Yue, Distributed adaptive consensus tracking for a class of multi-agent systems via output feedback approach under switching topologies, Neurocomputing 174 (2016) 1125-1132. 


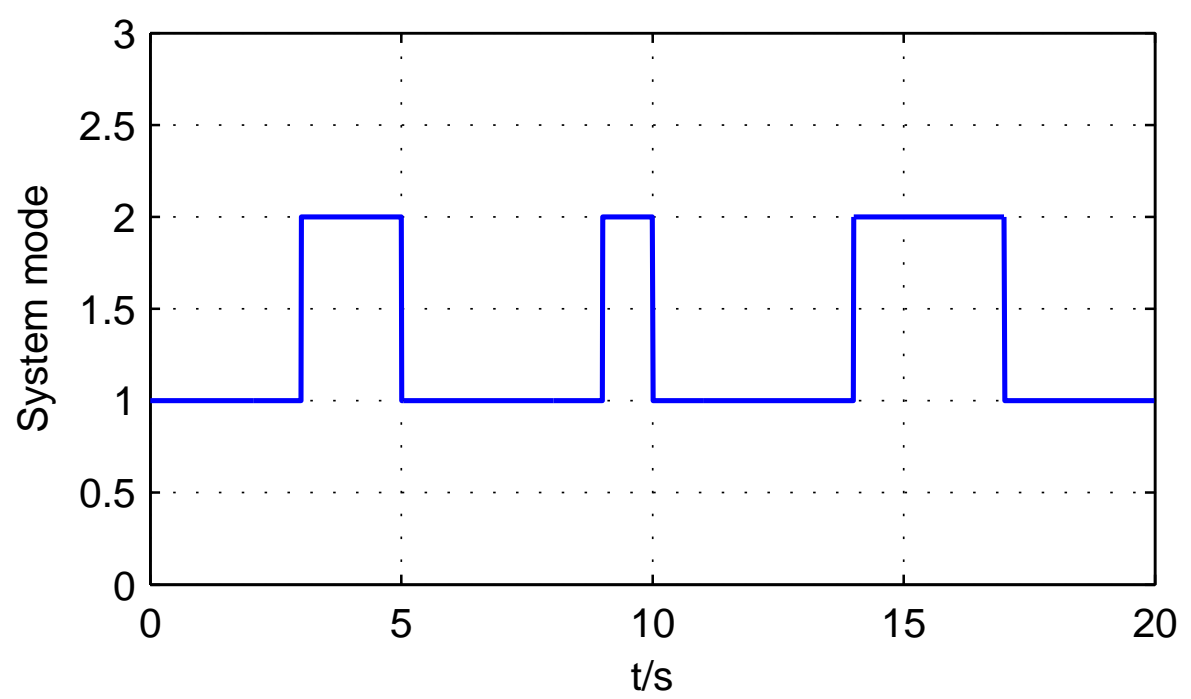

Figure 8: Switching signal

[5] W. Yang, S. Tong, Output feedback robust stabilization of switched fuzzy systems with time-delay and actuator saturation, Neurocomputing 164 (2015) 173-181.

[6] L. Long, J. Zhao, Adaptive fuzzy tracking control of switched uncertain nonlinear systems with unstable subsystems, Fuzzy Sets and Systems 273 (2015) 49-67.

[7] B. Niu, Z. Xiang, State-constrained robust stabilisation for a class of high-order switched nonlinear systems, IET Control Theory and Applications 9 (2015) 1901-1908.

[8] M. L. Chiang, L. C. Fu, Adaptive stabilization of a class of uncertain switched nonlinear systems with backstepping control, Automatica 50 (2014) 2128-2135.

[9] M. Hou, F. Fu, G. Duan, Global stabilization of switched stochastic nonlinear systems in strict-feedback form under arbitrary switchings, Automatica 49 (2013) 2571-2575.

[10] Z. J. Wu, X. J. Xie, P. Shi, Y. Q. Xia, Backstepping controller design for a class of stochastic nonlinear systems with Markovian switching, Automatica 45 (2009) 997-1004.

[11] H. Li, P. Shi, D. Yao, L. Wu, Observer-based adaptive sliding mode control for nonlinear Markovian jump systems, Automatica 64 (2016) 133-142.

[12] H. Li, H. Gao, P. Shi, X. Zhao, Fault-tolerant control of Markovian jump stochastic systems via the augmented sliding mode observer approach, Automatica 50 (7) (2014) 1825-1834.

[13] X. Zheng, X. Zhao, R. Li, Y. Yin, Adaptive neural tracking control for a class of switched uncertain nonlinear systems, Neurocomputing 168 (30) (2015) 320-326.

[14] X. Zhao, X. Zheng, B. Liu, L. Liu, Adaptive tracking control for a class of uncertain switched nonlinear systems, Automatica 52 (2015) 185-191.

[15] B. Zhu, T. Zhang, Robust adaptive control for a class of switched nonlinear systems in pure-feedback form, Proceedings of the 2012 International Conference on Machine Learning and Cybernetics 3 (2012) 851-856.

[16] S. Huang, Z. Xiang, Adaptive finite-time stabilization of a class of switched nonlinear systems using neural networks, Neurocomputing 173 (2015) 2055-2061.

[17] M. Cai, Z. Xiang, Adaptive neural finite-time control for a class of switched nonlinear systems, Neurocomputing 155 (2015) 177-185.

[18] M. Cai, Z. Xiang, Adaptive fuzzy finite-time control for a class of switched nonlinear systems with unknown control coefficient, Neurocomputing 162 (2015) 105-115.

[19] Q. Zhou, P. Shi, Y. Tian, M. Wang, Approximation-based adaptive tracking control for MIMO nonlinear 
systems with input saturation, IEEE Transactions on Cybernettcs 45 (10) (2015) 2119-2118.

[20] Y. Hou, S. Tong, Adaptive fuzzy output feedback control for a class of nonlinear switched systems with unmodeled dynamics, Neurocomputing 35 (2015) 85-93.

[21] S. Tong, S. Sun, Y. Li, Fuzzy adaptive output feedback control of MIMO nonlinear systems with partial tracking errors constrained, IEEE Transactions on Fuzzy Systems (23) (4) (2015) 729-742.

[22] Y. Li, S. Tong, T. Li, Observer-based adaptive fuzzy tracking control of MIMO stochastic nonlinear systems with unknown control direction and unknown dead-zones, IEEE Transactions on Fuzzy Systems 23 (4) (2015) 1228-1241.

[23] T. Wang, H. Gao, J. Qiu, A combined adaptive neural network and nonlinear model predictive control for multirate networked industrial, IEEE Transations on Neural Networks and Learning Systems (27) (2) (2016) 416-425.

[24] C. M. Lin, C. S. Hsueh, C. H. Chen, Robust adaptive backstepping control for a class of nonlinear systems using recurrent wavelet neural network, Neurocomputing 74 (2010) 481-486.

[25] X. Wang, T. Li, C. L. Philip. Chen, B. Lin, Adaptive robust control based on single neural network approximation for a class of uncertain strict-feedback discrete-time nonlinear systems, Neurocomputing 138 (2014) 325-331.

[26] M. Krstic, I. Kanellakopoulos, P. V. Kokotovic, Nonlinear and Adaptive Control Design, Wiley, NewYork, 1995.

[27] Y. J. Liu, S. Tong, Adaptive fuzzy control for a class of unknown nonlinear dynamic systems, Fuzzy Sets and Systems 263 (2015) 49-70.

[28] Q. Wang, C. Wen, Decentralized robust adaptive output feedback control of stochastic nonlinear interconnected systems with dynamic interactions, Automatica 54 (2015) 124-134.

[29] T. Zhang, X. Xia, Adaptive output feedback tracking control of stochastic nonlinear systems with dynamic uncertainties, International Journal of Robust and Nonlinear Control 25 (2015) 1282-1300.

[30] T. Zhang, X. Xia, Decentralized adaptive fuzzy output feedback control of stochastic nonlinear largescale systems with dynamic uncertainties, Information Sciences 315 (2015) 17-38.

[31] X. Xia, T. Zhang, Decentralized adaptive output feedback dynamic surface control of interconnected nonlinear systems with unmodeled dynamics, Journal of the Franklin Institute 352 (2015) 1031-1055.

[32] S. Yin, P. Shi, H. Yang, Adaptive fuzzy control of strict-feedback nonlinear time-delay systems with unmodeled dynamics, IEEE Transactions on Cybernetics (2015) DOI: 10.1109/TCYB.2015.2457894.

[33] X. Xia, T. Zhang, Adaptive output feedback tracking control of nonlinear systems with input unmodeled dynamics and prescribed performance, Proceedings of the 27th Chinese Control and Decision Conference (2015) 471-476.

[34] J. Chen, T. Zhang, X. Xia, Q. Wan, Adaptive output feedback control of nonlinear systems with input unmodeled dynamics, Proceedings of the 27th Chinese Control and Decision Conference (2015) 1303-1308.

[35] J. Chen, T. Zhang, X. Xia, Output feedback adaptive control of systems with input and state unmodeled dynamics, Control and Decision 10 (2015) 1847-1853.

[36] M. Z. Hou, A. G. Wu, G. R. Duan, Robust output feedback control for a class of nonlinear systems with input unmodeled dynamics, International Journal of Automation and Computing 5 (3) (2008) 307-312.

[37] M. Krstic, J. Sun, P. V. Kokotovic, Control of feedback linearizable systems with input unmodeled dynamics, Proceedings of the 33rd IEEE Conference on Decision and Control 2 (2) (1994) 1633-1638.

[38] M. Krstic, J. Sun, P. V. Kokotovic, Robust control of nonlinear systems with input unmodeled dynamics, IEEE Transactions on Automatic Control 41 (6) (1996) 913-920.

[39] W. Zha, J. Zhai, S. Fei, Y. Wang, Finite-time stabilization for a class of stochastic nonlinear systems via output feedback, ISA Transactions 53 (2015) 709-716.

[40] K. Lu, Y. Xia, Adaptive attitude tracking control for rigid spacecraft with finite-time convergence, Automatica 49 (12) (2013) 3591-3599.

[41] Y. Zhao, Z. Duan, G. Wen, G. Chen, Distributed finite-time tracking for a multi-agent system under a leader with bounded unknown acceleration, Systems and Control Letters 81 (2015) 8-13.

[42] S. P. Bhat, D. S. Bernstein. Finite-time stability of continuous autonomous systems, SIAM Journal on 
Control and Optimization 38 (3) (2000) 751-766.

[43] L. Wang, Adaptive Fuzzy Systems and Control: Design and Stability Analysis, Prentice Hall, NJ, 1994.

[44] C. Qian, W. Lin, Non-Lipschitz continuous stabilizers for nonlinear systems with uncontrollable unstable linearization, Systems and Control Letters 42 (3) (2001) 185-200.

[45] H. Li, Y. Gao, P. Shi, H. Lam, Observer-based fault detection for nonlinear systems with sensor fault and limited communication capacity, IEEE Transactions on Automatic Control (2015) DOI: 10.1109/TAC.2015.2503566.

[46] S. Yin, Z. Huang, Performance monitoring for vehicle suspension system via fuzzy positivistic C-means clustering based on accelerometer measurements, IEEE Transactions on Mechatronics 20 (5) (2015) 2613-2620.

[47] S. Yin, X. Zhu, O. Kaynak, Improved PLS focused on key-performance-indicator-related fault diagnosis, IEEE Transactions on Industrial Electronics 62 (3) (2015) 1651-1658.

\section{Biography of the authors}

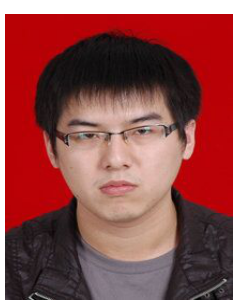

Jun Mao was born in Jiangsu Province, China, in 1988. He received his MS degree in Control Theory and Control Engineering from Yangzhou University, Yangzhou, China, in 2015. Now he is pursuing a PhD degree in Control Theory and Control Engineering from NUST, Nanjing, China. His current research interests include finite-time control, adaptive control, nonlinear systems and switched systems.

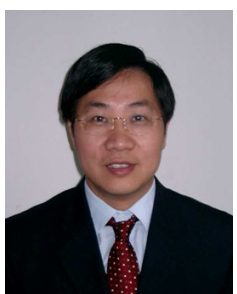

Zhengrong Xiang received his Ph.D. degree in Control Theory and Control Engineering at Nanjing University of Science and Technology, Nanjing, China, in 1998. Since 1998 he has been faculty member and he is currently full professor at Nanjing University of Science and Technology. He was appointed as Lecturer in 1998 and Associate Professor in 2001 at Nanjing University of Science and Technology. He is a member of the IEEE, member of the Chinese Association for Artificial Intelligence. His main research interests include switched systems, nonlinear control, robust control, and networked control systems.

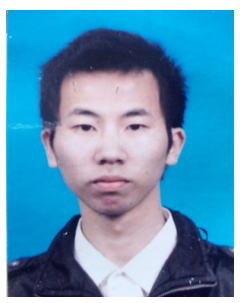

Shipei Huang received his B.S. degree in Mechanical and Electrical Engineering from Zhejiang Ocean University, Zhoushan, China, in 2011. He is currently pursuing his Ph.D. degree in Control Theory and Control Engineering at School of Automation, Nanjing University of Science and Technology, China. His research interests include nonlinear systems, switched systems and two-dimensional systems. 